
des personnes en mouvement | 2019

\title{
Circulations et quêtes thérapeutiques en santé mentale au Sénégal
}

\section{Véronique Petit}

\section{(2) OpenEdition}

Journals

Édition électronique

URL : http://journals.openedition.org/rfst/374

DOI : $10.4000 /$ rfst.374

ISSN : 2492-3672

Éditeur

Espaces et SOciétés (UMR 6590)

Référence électronique

Véronique Petit, «Circulations et quêtes thérapeutiques en santé mentale au Sénégal », Revue francophone sur la santé et les territoires [En ligne], Les circulations en santé : des produits, des savoirs, des personnes en mouvement, mis en ligne le 16 décembre 2019, consulté le 06 avril 2021. URL : http://journals.openedition.org/rfst/374 ; DOl : https://doi.org/10.4000/rfst.374

Ce document a été généré automatiquement le 6 avril 2021.

\section{(c) (i) (3) (2)}

La Revue francophone sur la santé et les territoires est mise à disposition selon les termes de la Licence Creative Commons Attribution - Pas d'Utilisation Commerciale - Partage dans les Mêmes Conditions 4.0 International. 


\title{
Circulations et quêtes thérapeutiques en santé mentale au Sénégal
}

\author{
Véronique Petit
}

\section{Introduction}

1 Cet article se propose de mettre en lumière certaines des circulations liées aux quêtes thérapeutiques qu'engagent des personnes malades, seules ou soutenues par «leur(s) accompagnant(s) » afin de remédier à une pathologie mentale. Ces cheminements ont été observés et interrogés dans le contexte du Sénégal, pays choisi en raison de l'introduction de la psychiatrie à l'époque coloniale, des dynamiques migratoires et des pratiques transnationales, et du pluralisme thérapeutique qui caractérisent cette société complexe. Le transnationalisme par les différents flux qui l'irriguent (hommes, produits, savoirs, pratiques, imaginaires), dessine les pratiques de santé et de soins (Dilger, Kane et Langwick, 2012). Le choix du recours des migrants peut être également influencé par leur appartenance sociale, qui peut être saisie à travers leurs origines, leur niveau d'éducation et leur activité professionnelle (Petit et Wang, 2019), mais aussi par les multiples actions des migrants dans leur société d'origine où souvent ils se substituent aux États défaillants en matière de santé et d'éducation (Kane, 2001). Si ces circulations ont déjà été mises en évidence dans d'autres contextes et à propos d'autres pathologies, elles demeurent insuffisamment documentées en ce qui concerne la santé mentale au Sénégal. Quelles que soient les disciplines, les recherches concernant la santé mentale en Afrique subsaharienne sont qualifiées de rares et pauvres, alors même que cette question émerge non sans difficulté sur la scène de la santé globale (Saxena et al., 2007).

2 Après des décennies de lobbying institutionnel et scientifique reposant sur l'idée qu'il est impossible de dissocier état sanitaire et santé mentale (Prince et al., 2007), cette dernière a été incluse dans les objectifs du développement durable (ODD) et dans la 
santé globale sortant ainsi de l'ombre où elle demeurait (Mnookin, 2016) (Patel et al., 2018). Une série de travaux épidémiologiques ont réévalué à la hausse le poids des maladies mentales dans la morbidité globale (Prince et al., 2007) (Funk, Benradia et Roelandt, 2014). Au niveau mondial, elles représentent en 2013 32,4\% des années vécues avec handicap et $13,0 \%$ des années de vie perdues, ce qui les situe juste après les maladies cardiovasculaires dans le calcul de la charge globale de morbidité (Global Burden of Disease Study 2013 Collaborators, 2015 ; Vigo, Thornicroft et Atun, 2016). Alors qu'elle apparait de plus en plus comme un enjeu sanitaire public au niveau international, la santé mentale ne s'impose toujours pas comme une priorité dans un grand nombre de pays qui sont dépourvus de politiques spécifiques dans ce domaine. C'est particulièrement vrai de l'Afrique puisque moins d'un quart des pays y disposent d'un programme en santé mentale (WHO, 2015).

3 Les troubles mentaux en tant que maladies non transmissibles ne constituent pas un péril sanitaire (Atlani-Duault et Vidal, 2013) d'autant que leurs conséquences sanitaires et socioéconomiques sont sous-estimées faute de mesure. Le manque de financements, la faiblesse des ressources accordées et l'indifférence politique au niveau national dont est l'objet la santé mentale sont soulignés de manière récurrente. Entre 1995 et 2015, sur 36 milliards de dollars alloués à la santé, seuls 110 millions ont été dépensés en faveur de la santé mentale (Charlson et al., 2017). L'absence d'implication politique est considérée comme un des freins majeurs à la reconnaissance de cette question sanitaire (Eaton et al., 2011), avec le manque de personnel qualifié et spécialisé, une centralisation excessive, l'absence de soins primaires de santé mentale et enfin la pénurie d'expertise en santé publique parmi les responsables de ce secteur sanitaire. L'inclusion dans la santé globale n'implique ni l'entrée dans une "ère de la générosité » (Garrett, 2007), ni l'émergence d'une mobilisation politique, institutionnelle et financière des différents acteurs de la santé globale.

4 Les personnes malades en particulier dans les pays en développement ne bénéficient donc pas de programmes ciblés et d'une prise en charge améliorée susceptible de modifier les recours et les traitements observés. Parmi les personnes ayant connu l'expérience d'une maladie mentale au cours de leur vie, 80 \% résident dans les pays en développement (Mnookin $2016: 3$ ). Alors qu'un nombre limité de médicaments suffit à traiter la majorité de ces pathologies, environ un quart des pays ne disposent pas des trois médicaments les plus fréquemment prescrits pour traiter la schizophrénie, la dépression et l'épilepsie au niveau des soins de santé primaires (Patel et al., 2007). Entre 75 et $85 \%$ des individus atteints de troubles mentaux graves dans ces pays n'y reçoivent aucun soin et ces personnes sont fréquemment atteintes dans l'exercice de leurs droits (Kastler, 2011). Les attitudes des populations vis-à-vis de la maladie mentale restent en effet encore largement déterminées par des croyances traditionnelles qui conduisent à des traitements inadaptés et à une stigmatisation des personnes malades (Gureje et Alem, 2000). Selon la Banque Mondiale (BM) et l'Organisation Mondiale de la Santé (OMS), la santé mentale n'est pas réductible à une simple question de santé du fait de ses multiples conséquences. Elle constitue en l'occurrence un enjeu de développement fondamental, car l'économie globale perd chaque année environ US $\$ 1$ trillion de productivité du fait des troubles mentaux (Kleinman et al., 2016).

5 Faute de mobilisation politique, les États africains demeurent dans l'impossibilité d'assurer des soins en santé mentale de qualité à destination de l'ensemble des 
personnes affectées et de mettre en œuvre les recommandations internationales concernant la décentralisation, l'inclusion dans la santé primaire et le respect des droits des malades. Faute de données épidémiologiques concernant les situations de base, les ressources, les évolutions des indicateurs ${ }^{1}$, il leur est difficile d'élaborer une politique de santé mentale efficace (Wone, 2018). Différentes raisons expliquent ce déficit d'informations statistiques en Afrique. Tout d'abord, la santé mentale n'est pas incluse dans les soins primaires. Les malades sont référés dans des centres spécialisés qui développent chacun leur base de données, ce qui complique le travail de compilation et de standardisation nécessaire à l'élaboration de séries statistiques cohérentes. Dans le cas du Sénégal, une procédure est actuellement en cours afin d'inclure les données de routine en santé mentale dans le système d'information sanitaire national à partir de la plateforme DHIS-2 (District Health Information Software). D'autre part, le travail de saisie informatique est réalisé par des agents de santé pour qui il représente un surcroit de travail et pour lequel ils ne sont pas toujours suffisamment formés et motivés (Ahanhanzo et al., 2015). Enfin, les indicateurs sont définis en fonction d'urgences et de partenariats décidés à un niveau international notamment dans le cadre des ODD ou du Fond Mondial, et non pas par rapport à des priorités définies localement (Hane, 2017). Dans le cas du Sénégal, l'inclusion de la santé mentale dans les ODD et l'obligation d'évaluer les progrès obtenus grâce à des indicateurs a déjà conduit à expérimenter un module « santé mentale » dans l'Enquête démographique et de santé continue (EDS-C) 2018 à notre initiative en partenariat avec l'Agence Nationale de Statistiques et de Démographie (ANSD).

6 Dans les pays en développement, face à l'iniquité et l'inefficacité du système de soins, les familles n'ont d'autres alternatives que d'assurer elles-mêmes la prise en charge des personnes atteintes de troubles psychiques (Saxena et al., 2007). Compte tenu de ces différents facteurs, nous avons considéré les malades et leurs parents comme des acteurs du travail médical et de la quête de soin entreprise dans la perspective développée par Anselm Strauss (Strauss et al., 1985). Dans le contexte sénégalais, les parcours en santé mentale, et les circulations qui les structurent, sont donc à replacer dans le contexte d'une offre psychiatrique insuffisante en dépit de son ancienneté, d'une couverture médicale universelle émergente et des représentations socioculturelles de la maladie mentale qui, nous en émettons l'hypothèse, impactent les choix des recours.

7 Le Sénégal constitue un observatoire heuristique des circulations liées à la quête de soins en santé mentale, en raison de l'histoire de la psychiatrie dans ce pays et de la place que les mobilités et migrations tiennent dans cette société. Ce pays dispose d'un système psychiatrique unique et reconnu à travers l'école de Fann héritée de la colonisation (Becker et Collignon, 1999; Collignon, 1999, 2002). Que ce soit dans l'empire colonial français, anglais ou hollandais, institutions et pratiques psychiatriques ont été disséminées dans une double perspective : offrir un recours aux colons et aux membres de l'administration coloniale souffrant de leurs nouvelles conditions de vie et contribuer à transformer les populations colonisées en une maind'œuvre performante à des travers des politiques de santé. 


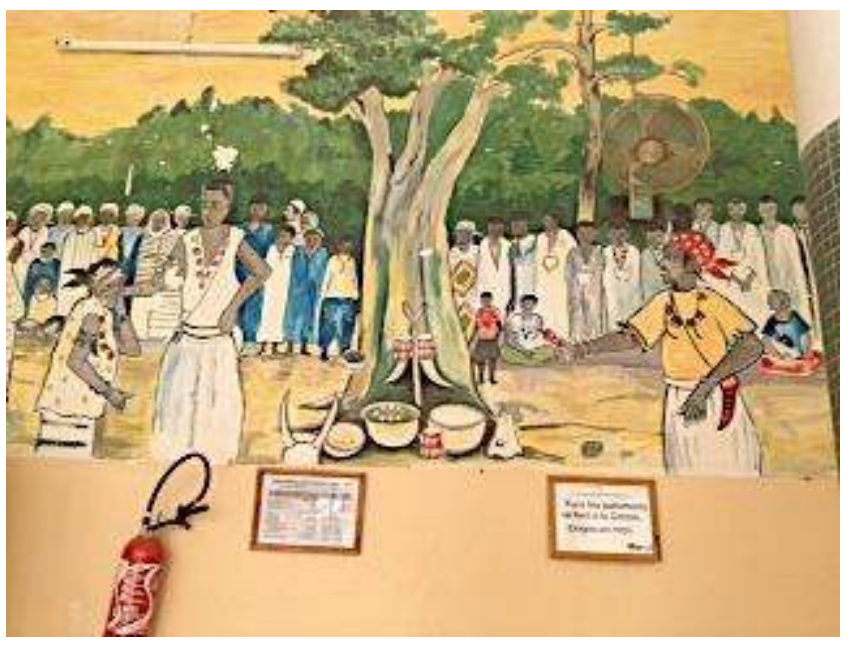

Cette fresque décrivant la cérémonie traditionnelle du ndoep en milieu lébou est située à l'intérieur de I'Hôpital psychiatrique de Thiaroye témoignant ainsi de l'approche culturelle développée par H. Collomb (Petit, 2013)

Photo : Petit, 2013

8 La France a mis en place l'assistance psychiatrique indigène en 1938 (API) après avoir constaté son retard par rapport à l'Angleterre et à la Hollande dans la prise en charge des maladies mentales dans leurs colonies. Les expérimentations psychiatriques conduites sur le continent se nourrissent les unes les autres, le psychiatre français Henri Collomb (1913-1979) s'inspire de l'expérience du village Aro élaborée par le psychiatre nigérian Thomas Tombo au Nigeria pour développer les villages thérapeutiques au Sénégal (Akyeampong, Hill et Kleinman, 2015) au début des années 1970. Des villages thérapeutiques sont implantés à Kénia en Casamance, à Djimkoré et à Botou vers Tambacounda dans le Sénégal oriental. Précédemment à partir de 1958, promouvant l'idée d'une psychiatrie sociale reconnaissant la culture de ses patients, Collomb inaugure de nouvelles pratiques thérapeutiques établissant un continuum entre l'hôpital et la société : l'accompagnant, le pénc ${ }^{2}$ et la collaboration avec des tradipraticiens [photographie 1]. Dans cette démarche compréhensive et visant à rapprocher deux systèmes de soin, il adjoint à son équipe médicale des anthropologues et sociologues. Ces essais thérapeutiques connaissent des destins divergents : le rôle actif de l'accompagnant demeure d'actualité, il connaît néanmoins de profondes transformations liés aux changements socioéconomiques et à l'évolution de l'organisation du système de santé (Diagne et Lovell, 2019; Koundoul, 2015). La pratique du pénc est inégale selon les contextes hospitaliers et tend à se distendre. Enfin, en raison de moyens suffisants et d'une faible adhésion des psychiatres nationaux dès leur création, les villages thérapeutiques n'ont pas perdurés (Collignon, 1983). La colonisation modifie en profondeur le système de soins en introduisant une nouvelle étiologie qui inaugure une étape décisive du pluralisme thérapeutique (Storper-Perez, 1974). Elle va également déstructurer les institutions des sociétés colonisées en les projetant avec violence dans la mondialisation. La mise en valeur des territoires par le biais des travaux forcés, du développement de l'économie de plantation et des cultures vivrières, du recrutement militaire, de l'urbanisation, provoque migrations internes et déplacements forcés. Aux mobilités séculaires s'ajoutent de nouvelles migrations internationales de travailleurs en particulier vers la 
métropole. L'indépendance ne met pas un terme à ces migrations qui prennent une place de plus en plus significative dans la société sénégalaise. À partir des années 1980, le système migratoire s'élargit et se structure grâce aux réseaux religieux, les routes et les profils migratoires se diversifient (Diop, 2010 ; Lessault, Robin et Georg, 2015). Le migrant devient une figure de réussite qui se substitue à celle du fonctionnaire et un acteur central du développement compte tenu de l'importance que les transferts prennent dans l'économie des familles et du pays. Ces mobilités constituent un substrat aux circulations thérapeutiques qui s'actualisent au fil des stratégies plurielles des acteurs en alimentant le pluralisme thérapeutique (Wang, 2019).

\section{Méthodes d'enquêtes et terrains}

\section{Questionnement initial}

9 Les données présentées ici sont issues d'une recherche conduite au Sénégal depuis 2016 à nos jours s'inscrivant dans une démarche de démographie anthropologique (Petit et al., 2019) (Petit, 2013). Elles prolongent un premier corpus collecté dans le cadre de l'ANR Sud MIPRIMO " La migration prise aux mots » (2011-2013) consacré à l'accès aux soins en santé mentale de migrants de retour au Sénégal (Ly, Petit et Pizzolato, 2014) et de populations majoritairement originaires d'Afrique de l'Ouest (Mali, Mauritanie, Guinée Conakry, Guinée Bissau). Il s'agissait alors d'analyser comment une maladie mentale pouvait interférer avec l'expérience d'une migration internationale. Ce questionnement initial a été élargi aux conditions d'accès aux soins psychiatriques en travaillant sur les parcours thérapeutiques, les modalités de prise en charge, les coûts des traitements et les représentations de la maladie chez la personne malade et dans son entourage. Cette enquête articule des données qualitatives et des données quantitatives.

\section{Espaces d'investigations}

10 En raison des comportements de stigmatisation et afin de saisir la dynamique des relations entre les différentes catégories d'acteurs, les services psychiatriques ont constitué des lieux d'investigation privilégiés. Plusieurs unités psychiatriques sur le territoire ont été sollicitées: dans la région de Dakar, la Clinique Moussa Diop, le service des consultations externes et le service de pédopsychiatrique Keur Xale Yi au CNHU de Fann à Dakar, l'Hôpital Psychiatrique de Thiaroye (service des urgences et service de pédopsychiatrie), dans la région de Thiès, le Centre de santé mentale Dalal Xel et ses consultations itinérantes à Louga et Diourbel, et le centre de santé mentale Émile Badiane à Ziguinchor en Casamance. D'autres services ont été visités dans l'objectif de mieux appréhender la diversité de l'offre. Si le secteur public et les centres catholiques ont constitué les principaux espaces de recherche en tant que structures historiques et celles accueillant le plus de patients, nos travaux s'orientent de plus en plus vers des cliniques ou des cabinets privés en psychiatrie et psychologie afin de tenir compte de l'évolution de l'offre de soin, de la complexité des parcours thérapeutiques et des carrières des acteurs concernés.

11 Les psychiatres que nous avons sollicités dans le cadre de ces travaux de recherche ont, sauf de très rares exceptions, répondu positivement à nos requêtes. Ils ont aussi parfois 
anticipé nos demandes ou spontanément proposés d'assister aux consultations, d'accéder aux documents médicaux, d'assister à des formations ou des activités organisées dans leurs services. Ils se sont presque tous systématiquement référés à la collaboration qu'avait instaurée Henri Collomb dans son équipe entre personnel médical et chercheurs en sciences sociales pour expliquer leur accueil positif s'inscrivant ainsi dans une forme de tradition de la psychiatrie culturelle sénégalaise.

\section{Les conditions de production des données discursives}

12 Les données discursives sont issues d'entretiens semi-directifs, de discussions informelles, d'observations et de suivis de consultations réalisés dans plusieurs services psychiatriques auprès des soignants, des malades et de leurs accompagnants. En effet, l'hospitalisation d'une personne malade au Sénégal se déroule conjointement avec celle de son accompagnant ${ }^{3}$. Ce dernier est un membre de la famille désignée par celle-ci ou une personne extérieure qui sera rétribuée pour remplir cette fonction. Dans ce cas, celle-ci est désignée de manière négative par le terme d'" accompagnant mercenaire " par les soignants. L'accompagnant a pour rôle, outre de s'occuper de la toilette et des repas du malade, de la propreté de la chambre, et surtout de servir d'interface entre le médecin et la famille afin de favoriser l'alliance thérapeutique. Dans le cadre de cette enquête, nous avons travaillé avec des accompagnants familiaux. Ce travail a été réalisé avec l'accord des autorités médicales et administratives afin de ne pas perturber l'activité médicale et respecter les droits des patients. Cinquante-sept entretiens semidirectifs ont été conduits ( 21 au CHU de Fann et l'HP de Thiaroye, 16 au centre de santé mentale de Ziguinchor, 20 au centre Dalal Xel de Thiès). Ceux-ci sont représentatifs des diverses strates sociales et de la diversité ethnique du pays, et sont quasiment également répartis en terme de sexe. Les entretiens ont été conduits en français ou avec l'aide d'un interprète formé en anthropologie de la santé.

13 Du côté des personnes malades et des accompagnants, les échanges informels et les entretiens d'orientation biographique ont constitué des temps d'expression appréciés au cours de journées marquées par l'ennui, l'attente et l'inquiétude. Les personnes sollicitées ou d'autres curieuses de notre présence, puis désireuses de partager leurs expériences, ont pu s'exprimer sans la crainte des jugements sociaux qui les obsèdent ordinairement. En effet, les psychiatres et les psychologues rencontrés ont souligné le poids de la honte ainsi que les mécanismes d'intériorisation du contrôle social dans la société sénégalaise, la peur de perdre la face. La folie suscite la peur et la répression. Les personnes victimes de troubles psychiques souffrent de discrédit, de perte d'autorité et sont marginalisées au sein de leurs familles et de la société. Elles sont l'objet de diverses formes de violences (contentions, rétention, déni de leurs droits, etc.), en particulier si les symptômes sont interprétés comme une transgression de l'ordre social, soit dans le sens d'un repli sur soi exagéré, soit dans celui d'une trop grande expressivité. Elles sont le plus souvent considérées comme un objet de honte par leurs parents en dépit des liens affectifs.

14 Nous avons pu conduire des entretiens sur plusieurs jours avec les personnes malades qui ont été rencontrées durant le temps de l'hospitalisation, en particulier dans le centre Emile Badiane à Ziguinchor et Dalal Xel à Thiès. En les retrouvant tous les deux ou trois jours, nous pouvions discuter avec elles et leurs accompagnants de l'évolution de la situation médicale, de leur appréciation des conditions de soins ainsi que de leurs

Revue francophone sur la santé et les territoires, Les circulations en santé : des produits, des savoirs, des personnes en mouvement 
difficultés sociales et financières face à la maladie. Ce passage régulier auprès des malades a permis de créer une relation de confiance plus assurée et de croiser différentes voix du récit familial autour de la quête thérapeutique. Cette relation a été prolongée avec une quinzaine de patients que nous avons revus à leur domicile ou dans d'autres lieux qu'ils jugeaient propices à la préservation de leur anonymat et dans lesquels ils se sentaient à l'aise. Certains d'entre eux nous ont proposé de les accompagner à des cérémonies religieuses telles que des séances d'exorcisme dans une église catholique à Thiès et lors de réunions de prières dans de nouvelles églises. Nous avons aussi enquêté auprès de guérisseurs et de marabouts réputés pour leur traitement des «fous » et de centres roqya à Dakar et Ziguinchor qui pratiquent « une médecine mystique» pour reprendre leur expression à travers les prescriptions coraniques. L'entrée dans ces espaces a permis d'observer des pratiques, des relations thérapeutiques et la mobilisation de produits et de savoirs d'origines diverses révélant certaines circulations qui traversent le quotidien des familles affectées par les troubles mentaux. C'est aussi une manière de connaître les "mondes sociaux » que traversent les acteurs, la porosité de ces univers et leurs ancrages.

\section{Approche quantitative}

La situation épidémiologique, les caractéristiques sociodémographiques des personnes malades et celles de leur ménage ne peuvent être décrites en détail faute de données statistiques suffisantes à l'échelle nationale. Les soins en santé mentale n'étant pas inclus dans les structures de santé primaire, les malades sont automatiquement référés dans les services spécialisés. Les structures psychiatriques publiques et privées créent leurs propres modalités d'enregistrement, mais leurs données ne sont pas fusionnées au niveau du Ministère de la Santé et de l'Action Sociale (MSAS). De plus, pour l'instant le MSAS n'a pas imposé l'utilisation d'une unique classification des maladies mentales, les services utilisent soit la classification internationale des maladies (OMS), une classification de l'INSERM adaptée au Sénégal ou le Manuel diagnostique et statistique des troubles mentaux (Diagnostic and Statistical Manual of Mental Disorders de l'Association américaine de psychiatrie). Cette pluralité de références est révélatrice des influences qui traversent la psychiatrie sénégalaise. La Direction du centre de santé mentale de Ziguinchor a mis à notre disposition ses fichiers de suivi de consultations, ceux-ci ont été anonymisés et analysés. Bien qu'ils n'offrent que peu de variables et qu'elles impliquent de nombreux de recodages en raison des circonstances de la saisie, ils procurent des informations relatives à l'origine des patients. Si nous avons pu consulter le fichier du centre catholique Dalal Xel de Thiès, nous n'avons pas été autorisés à développer une analyse statistique à partir de cette base de données alors qu'elle est d'une profondeur temporelle significative et d'une grande richesse sur les itinéraires thérapeutiques et les ressources des patients.

\section{Offre psychiatrique et parcours thérapeutiques}

Dans cette partie nous allons examiner comment l'offre psychiatrique se positionne dans les parcours thérapeutiques et comment elle contribue à structurer les circulations des personnes malades. En modifiant le système d'interprétation des désordres mentaux, l'institution psychiatrique a transformé la tolérance traditionnelle à l'égard du fou en un rejet. «Il [le fou] tend à devenir comme en Europe, objet 
d'incompréhension et de mépris. Il faut s'en préserver. Familles, voisins, collègues et employeurs commencent à rejeter cet être dangereux qu'il faut cacher derrière les murs de l'hôpital. Ces nouvelles attitudes sont en relation avec les structures sociales actuelles et avec l'institution psychiatrique." (Storper-Perez, 1974). L'institution psychiatrique ayant été utilisée tant par la puissance coloniale que par le jeune Etat indépendant comme un outil de répression sociale (Collignon, 1984) (Diop, 1997), le souvenir des rafles des populations marginales internées à Thiaroye reste vivace dans la mémoire collective et marque négativement l'institution psychiatrique.

\section{Une offre psychiatrique difficilement accessible}

17 Outre les barrières sociales, l'inégale répartition de l'offre de soins en santé mentale sur le territoire et l'éloignement des populations qu'elle implique retarde l'entrée dans les services psychiatriques. Certaines régions comme celles de Matam et Kédougou sont totalement dépourvues de structure de soins, d'autres disposent uniquement de consultations itinérantes réalisées par des équipes thérapeutiques provenant d'autres centres de santé mentale. Ainsi un des psychiatres du centre catholique Dalal Xel se rend à Diourbel et à Louga une fois par mois, alors que cette consultation devait être assurée par le médecin de l'hôpital régional de Saint-Louis. Les centres Dalal Xel de Thiès et Fatick constituent une sorte de sas de protection de la région de Dakar puisqu'ils reçoivent des populations de tout le nord et nord-est du pays, à tel point que la direction de ces institutions envisage de créer une troisième consultation à Matam ou Richard-Toll afin de faire face à l'afflux de malades originaires des régions frontalières. Le centre Emile Badiane à Ziguinchor est la seule structure psychiatrique de Casamance. Il assure aussi une consultation mensuelle à Kolda. Certaines structures n'offrent que quelques lits (l'hôpital de Saint-Louis par exemple), ou elles peinent à trouver un personnel qualifié désireux de travailler dans une région excentrée ou dont elles ne sont pas originaires. Tel est le cas du centre de Djimkoré à Tambacounda réhabilité après plusieurs années de fermeture. Par contre, Dakar concentre l'essentiel des ressources humaines et matérielles en santé mentale [carte 1] comme dans les autres spécialités.

À ce déséquilibre entre les régions sur la façade atlantique et les régions du centre et de l'est du pays se superpose une insuffisance de l'offre. Selon les chiffres de la Division de la Santé Mentale du MSAS, 36 psychiatres exercent en 2018 au Sénégal. Seulement sept d'entre eux (soit 15,2\%) sont affectés à l'extérieur de la région de Dakar. La population du Sénégal compte 15256346 individus en 2017 dont 53,5\% résident en milieu rural. La région de Dakar représente $23 \%$ de la population, suivi par les régions de Thiès $(13,1 \%)$ et de Diourbel (11\%) (ANSD, 2018). De manière classique en Afrique subsaharienne, on constate un déséquilibre entre l'offre de soin concentrée dans la région de la capitale au détriment du monde rural. Le ratio au Sénégal est de 0,26 psychiatre pour 100000 habitants. En réalité, ce ratio est encore plus faible $(0,18)$ puisque huit psychiatres sur 36 sont affectés à la santé des fonctionnaires à Hôpital militaire Principal à Dakar. Compte tenu du coût des prestations dans cette structure hospitalière et du fait qu'il faille déposer une caution avant d'être soigné, une large partie de la population du pays n'a pas accès à cette structure renommée pour la qualité de ses prestations. Selon l'OMS, il y a en moyenne 6,6 psychiatres pour 100.000 habitants dans les pays à hauts revenus, contre moins de 0,5 pour les pays à revenu faible moyen-inférieur (WHO, 2015). Au regard de ces moyennes qui cachent de profondes disparités, l'offre en santé 
mentale du Sénégal n'apparaît pas comme la plus dramatique au sein des pays en développement. Aminata Niang Diene dans son étude sur la gouvernance de la santé au Sénégal a effectué une cartographie de l'offre de soin spécialisé, elle révèle ainsi que la situation de la santé mentale illustre le « contraste saisissant » entre l'Ouest et le reste du pays où l'offre est déficitaire et incomplète : « La spécialisation médicale renforce la concentration des médecins dans les centres urbains de l'Ouest, notamment dans la capitale sénégalaise. (...) Fortement défavorisées, les autres régions sont soumises à la double contrainte d'une offre médicale éloignée et d'un déficit de médecins et de plateaux techniques spécialisés.»(Niang Diene 2019, 466). Si d'autres spécialités comme la cancérologie, sont dans une configuration encore moins favorable que la psychiatrie, il faut rappeler qu'elles correspondent à des enjeux sanitaires plus récents et qu'elles ne disposent pas de la profondeur historique de l'implantation de la psychiatrie. L'inégalité sociale et spatiale dans l'accès aux soins est inscrite dans la structure de l'offre de soin actuelle malgré la modification de la carte sanitaire et la création de nouveaux districts sanitaires qui visent à améliorer la qualité et la proximité de cette offre dans le cadre de la décentralisation.

Carte 1 : Répartition de l'offre en santé mentale au Sénégal (2019)

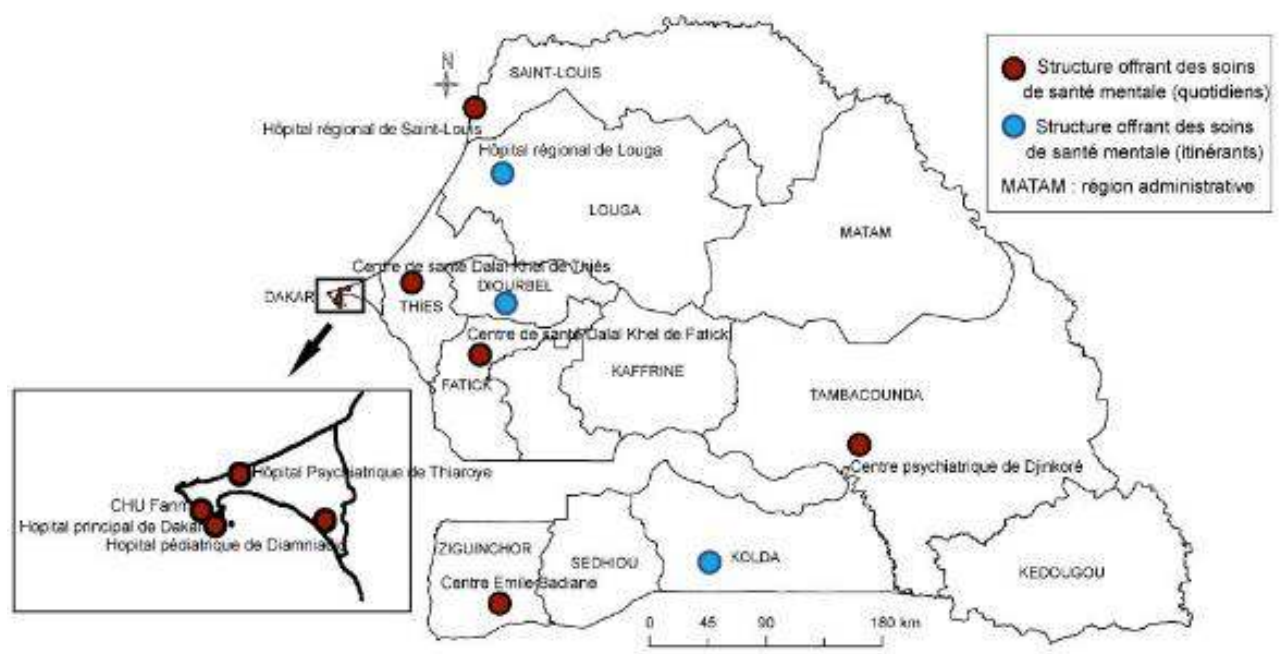

Réalisation : Aminata Niang Dieng, Dakar, Université Cheik Anta Diop, département de géographie, 2019

L'offre de santé mentale au Sénégal en dépit de son caractère inégal et insuffisant, constitue néanmoins une offre attractive au niveau de la sous-région, ce qui relativise la situation sanitaire du Sénégal. En effet, les pays frontaliers du Sénégal (Mauritanie, Mali, Guinée Bissau, République de Guinée, Gambie) ne disposent pas d'une offre de santé mentale ou à proximité des populations concernées en raison de l'état de leur système de santé lié et du contexte politique et économique parfois fortement dégradé. Aussi les centres de santé mentale reçoivent également des patients étrangers et tout particulièrement des États limitrophes. Les centres de Thiès et Tambacounda reçoivent par exemple des populations du Mali en raison de leurs situations sur les axes routiers entre Bamako et Dakar. Commerçants et camionneurs profitent de leurs déplacements professionnels pour consulter tout en préservant ces visites médicales secrètes par rapport à leur famille. L'effet de proximité avec la frontière est encore plus visible en ce qui concerne la région de Ziguinchor puisque presque $12 \%$ des malades qui consultent 
au centre Émile Badiane proviennent des pays frontaliers [tableau 1]. Les groupes ethniques qui vivent en Casamance circulent dans une aire culturelle et linguistique qui s'ancre dans des pratiques économiques coutumières, une histoire sociale profonde qui ne correspond pas aux découpages nationaux actuels, et des réseaux de parenté transnationaux. Si des patients étrangers viennent consulter au centre Emile Badiane, d'autres malades traversent de part et d'autre la frontière afin de se rendre chez des marabouts et des guérisseurs réputés pour remédier à la folie. Les récits recueillis montrent que fréquemment dans ces zones rurales les personnes malades conduites sont conduites en situation de crise au centre de santé mentale. Auparavant, les malades sous l'impulsion de leurs parents expérimentent une succession de tradithérapeutes recommandés par leur entourage familial et leur voisinage ou dont les échos de leur renommée leur parviennent. La décision de se rendre en psychiatrie n'est prise qu'à reculons tant la honte et la stigmatisation associées à la folie sont fortes et indélébiles. Inversement se rendre chez un guérisseur est une pratique socialement reconnue et acceptée par tous, elle n'implique pas de facto une association avec la folie. Elle s'inscrit dans la normalité des pratiques de soins et une étiologie partagée contrairement à celle de la psychiatrie.

Tableau 1 : Répartition des personnes malades ayant consulté à Ziguinchor selon leur lieu de résidence (calculs pour 2017)

\begin{tabular}{|l|l|l|}
\hline Lieu de résidence & Effectifs & Pour cent \\
\hline Régions du Sénégal & & \\
\hline Dakar & 107 & 0,48 \\
\hline Diourbel & 3 & 0,01 \\
\hline Fatick & 10 & 0,05 \\
\hline Kaffrine & 4 & 0,02 \\
\hline Kaolack & 3 & 0,01 \\
\hline Kolda & 941 & 4,25 \\
\hline Louga & 5 & 0,02 \\
\hline Matam & 5 & 0,02 \\
\hline Saint-Louis & 5 & 0,02 \\
\hline Sedhiou & 2984 & 13,48 \\
\hline Tambacounda & 15 & 0,07 \\
\hline Thiès & 28 & 0,13 \\
\hline Ziguinchor & 15139 & 68,4 \\
\hline Résidence inconnue & 81 & 0,37 \\
\hline Pays frontaliers & & \\
\hline Cap vert & 2 & 0,01 \\
\hline Gambie & 921 & 4,16 \\
\hline Guinée Bissau & 1667 & 7,53 \\
\hline Mali & 8 & 0,04 \\
\hline République de Guinée & 2 & 0,01 \\
\hline ND & 53 & 0,24 \\
\hline Total & 22132 & 100 \\
\hline & & \\
\hline
\end{tabular}

Source : Centre Émile Badiane, Ziguinchor 2018

\section{La psychiatrie, un recours contraint et subi}

Les personnes ne résidant pas dans les régions de Dakar et de Thiès doivent effectuer de longs déplacements afin de se rendre dans un centre psychiatrique. Les malades, seuls ou accompagnés, font des trajets de plusieurs centaines de kilomètres engendrant 
des coûts de transport, d'hébergement et de restauration non négligeables auxquels s'ajoutent les frais médicaux (consultations, examens, analyses, traitements). Le coût de l'hospitalisation varie selon les services et les conditions de séjour proposées.

21 Dans les centres de santé mentale en zone rurale (Thiès, Ziguinchor) on peut estimer qu'une famille pourra dépenser 10.000 à 20.000 FCFA pour un malade de manière journalière. Lorsqu'un malade est hospitalisé, la règle est qu'il soit gardé au minimum dix jours en observation afin d'établir un diagnostic, selon la gravité de la pathologie la durée d'hospitalisation sera plus ou moins prolongée. Les entretiens révèlent que les familles les plus vulnérables sont contraintes de mobiliser leurs réseaux afin de réunir les fonds nécessaires au règlement des soins, elles s'endettent auprès de leurs parents, migrants urbains et internationaux, amis et voisins, elles peuvent être amenées à vendre certains de leurs biens (bijoux, récoltes, bêtes). Le centre Emile Badiane et le centre Dalal Xel ont mis en place des politiques de crédit afin que les familles puissent payer les soins de manière étalée et différée, afin de garantir l'accessibilité aux soins dans ces zones rurales. Certaines familles dont la structure familiale est resserrée (par exemple des familles monoparentales composée d'une mère et de ses enfants, des parents âgés avec des enfants ou petits-enfants) voient l'économie du ménage fortement ébranlée par le coût de la maladie, et ce, d'autant plus si le malade fait partie des actifs productifs. Dans ces régions, rares sont les familles qui disposent d'une mutuelle de santé parfois elles n'ont même pas entendu parler de ce concept ${ }^{4}$. D'autres, disposant d'une couverture maladie, se plaignent des dysfonctionnements de leur assurance: retard dans les remboursements, médicament non remboursés, désaccords avec les pharmacies. Cette charge financière peut conduire lorsque les parents pensent que la personne malade va mieux à l'interruption du traitement ou à des aménagements de la prescription médicamenteuse (la posologie est fractionnée ou réduite, on demande au médecin quel est le médicament essentiel afin de ne pas acheter tous les médicaments). À l'anxiété et au désarroi provoqués par la maladie, viennent s'ajouter chez les parents de la personne malade le stress lié à leur incapacité d'assumer cette dépense.

22 Tant qu'elles le peuvent, les familles évitent de consulter un service psychiatrique qui les assigne à la folie de la personne malade dans le contexte social. Afin d'éviter la honte et le déshonneur, les parents responsables du malade cherchent à préserver les apparences grâce à des recours qui évitent toute association avec la psychiatrie. Ils ne se rendent dans un service psychiatrique qu'après avoir expérimenté les recours auxquels ils ont accès et dont ils ont connaissance durant des années (marabouts, guérisseurs, prêtes exorcistes, séances de prières de nouvelles églises). Ils ne s'y résolvent que face à l'aggravation de l'état du malade ou une crise qui les laissent démunis : "Si on est à Dakar et si on a la chance d'avoir un parent "intellectuel" on va envoyer le malade à Fann. Il va avoir de la chance. Les choses évoluent. Autrement on va le conduire chez un marabout, puis de marabout en marabout parce que cela ne va pas mieux. Puis le parent parfois est délaissé. On dit que le traitement des Blancs ne peut rien contre les djinns, les rab ${ }^{5}$ " (FM, interne en psychiatrie Fann, 2016). L'entrée en psychiatrie se concrétise parfois brutalement lorsque l'entourage familial ou le voisinage requière l'intervention du chef de quartier ou de village, des pompiers, de la police ou de la gendarmerie face à des comportements qui ne sont pas socialement tolérés comme par exemple des agressions physiques entrainant des blessures, entrer par effraction chez des voisins, des comportements exhibitionnistes. 
23 Dans ce contexte tendu, la première hospitalisation, le déroulement des premières consultations et les effets constatés du traitement sont déterminants afin que s'enclenche un suivi médical continu. L'adhésion au traitement est un processus incluant abandons, rechutes, crises, retours, phases de stabilisation. Certains malades s'ils sont satisfaits de l'alliance thérapeutique qui se met en place avec le psychiatre (évolution de santé, écoute, confiance), font leur possible pour préserver cette relation dans le cas où le praticien change d'affectation, passant d'une région à une autre, d'une structure à une autre ou du secteur public au secteur privé. Les personnes malades traversent aussi des phases d'errance thérapeutique. Les patients dans leur grande majorité ne disposent ni d'une activité professionnelle ni de revenus du fait de leur état de santé. Le financement de leurs traitements et le type de recours choisi dépendent de l'implication de leurs parents. Face à l'étirement du coût du traitement psychiatrique sur des mois, des années, voire définitivement, nombre de parents sont tentés de suspendre le traitement lorsqu'ils jugent que le malade se conforme à leurs attentes sociales, provoquant ainsi une rechute suivie d'une nouvelle phase d'expérimentations thérapeutiques. " Ma mère m'a jeté sur la route la nuit pour aller voir un marabout qu'une voisine lui a recommandé. J'ai marché tout seul, pris des taxis, avec un peu d'argent que mon père m'a donné et mes affaires. J'ai traversé la moitié du Sénégal. Pour rien. Elle ne voulait plus payer mon ordonnance. Moi je voulais continuer l'ordonnance. C'est ça qui m'aide » témoigne FS (38 ans, électricien sans emploi, serer, village à proximité de Thiès). Cet extrait met en exergue les divergences qui peuvent exister entre la personne souffrante et ses proches dans le choix des recours thérapeutiques. Ici, le malade souhaite se rendre dans le centre santé mentale et poursuivre le traitement thérapeutique qu'il entamé et dont il mesure les effets positifs, d'où sa référence à l'ordonnance qui symbolise à la fois les médicaments et à la nécessité de respecter la posologie sur le long terme.

\section{La distance comme stratégie de protection}

L'éloignement entre le lieu de résidence du malade et le service psychiatrique où il consulte s'explique aussi par la peur associée au discrédit social qu'entraîne l'assignation à la folie. Une psychiatre du CHU de Fann rapporte que des visiteurs au sein de l'hôpital lui ont adressé la requête suivante : «Si vous pouviez attacher les malades à un piquet où vous voulez, même dans l'hôpital, et ne plus les laisser sortir ça serait mieux, qu'on ne les voit plus, ne les entende plus ». Familles et patients, en particulier s'ils disposent d'un statut social élevé, souhaitent garder leur état de santé le plus secret possible. Ils craignent d'être disqualifiés par effet de contagion dans l'ensemble de leurs cercles de sociabilité (social, familial, professionnel, politique) par la suspicion et la rumeur. L'anonymat que procure la distance devient alors une condition du soin. Le processus de distanciation dépend du capital et des ressources qui peuvent être mobilisées autour de la personne malade. La mise à distance s'opère à plusieurs échelles : consulter dans une autre ville ou une autre région que celle où l'on réside, voyager à l'étranger, profiter de déplacements professionnels, se rendre dans une consultation privée, solutions qui sont jugées plus discrètes qu'une consultation dans un service hospitalier. La répartition spatiale de l'offre en santé mentale ne doit donc pas être lue uniquement de manière négative comme un frein à l'accessibilité, la carte des districts sanitaires peut être envisagée comme un territoire sur lequel les acteurs recherchent des solutions pragmatiques à leurs problèmes de santé. Comme le souligne Di Méo « (...) entre les territorialités ${ }^{6}$ et de tels territoires politiques, une tension 
s'établit dans l'ordre de la pratique comme dans celui des représentations et de la cognition. Cette tension s'affirme en tant que facteur majeur, tantôt frein, tantôt moteur, de l'action humaine et sociale spatialisée » (Di Méo, 2006). Les individus y déploient des stratégies en s'appuyant sur leurs ressources, leurs compétences et leurs motivations afin d'accéder aux soins désirés en préservant leur respectabilité et en s'individualisant encore davantage.

25 Le recueil de nos données concernant les personnes malades qui se soignent à l'étranger en particulier en Europe où elles disposent de réseaux et de ressources doit être complété. Nous nous sommes davantage attachés aux mobilités nord-sud de migrants internationaux qui revenaient se faire soigner au Sénégal après avoir initié un traitement psychiatrique dans leur pays d'immigration en Europe ou en Amérique du Nord. Ces retours thérapeutiques au pays d'origine sont parfois contraints par la situation de vulnérabilité dans laquelle se trouvent les migrants malades en Europe ou en Amérique du Nord et ils répondent à une demande des familles restées au pays. Dans un premier le temps le statut de migrant " very very VIP " permet d'accéder plus directement à la psychiatrie. Les parents, surtout si le migrant a déjà été en contact avec des soins psychiatriques ou s'ils disposent d'exemples de malades soignés ainsi dans leur milieu, considèrent que la psychiatrie est la solution la plus efficace afin de restaurer rapidement la santé du migrant en souffrance psychique. Il s'agit de rétablir l'ordre social et de préserver les apparences en "faisant taire» le malade s'il est en proie à des délires ou des gestes désordonnés; ensuite, certains psychiatres mentionnent qu'ils doivent faire face à des exigences pressantes de la part des parents afin qu'ils prescrivent un traitement autorisant le migrant à reprendre le plus rapidement les activités professionnelles qui lui permettent d'effectuer des transferts financiers vers les siens. Ces médecins soulignent le déni des familles quant au coût psychique de la migration (Ly, Petit et Pizzolato, 2014). On assiste donc à un nouvel investissement sur le migrant, après avoir constitué un capital initial permettant le départ en migration, le groupe se mobilise une seconde face à la nécessité de rétablir l'intégrité psychique du migrant. Néanmoins, il s'effrite progressivement face à la durée des traitements et à l'impossibilité de remigrer et d'entretenir ainsi la rente migratoire (Petit, 2019). La fabrique des soins s'analyse au niveau méso auquel sont prises les décisions (familles, réseaux) et dans le cadre de l'économie morale sur laquelle repose la migration internationale. Certains migrants installés en Europe suivent une psychothérapie à distance grâce à des consultations par Skype. Ces rendezvous concernent également la santé des conjoints et des enfants, tous n'étant pas migrants. Cette pratique cumule plusieurs avantages: la réduction des coûts, des échanges jugés plus productifs avec un clinicien sénégalais décrit comme percevant mieux les croyances et l'organisation de la parenté, la possibilité de s'exprimer dans sa langue. Cette invisibilité préserve l'image des migrants au sein de la diaspora et de leur famille au pays, ce qu'ils considèrent être un bénéfice substantiel. Ce type de pratique implique que la personne malade soit déjà avancée dans l'éducation thérapeutique et qu'elle ait acquis les compétences nécessaires à l'autogestion de sa maladie et au suivi de ses traitements. Les familles qui disposent de migrants internationaux dans leur parenté ont la possibilité d'accéder aux traitements de dernière génération pas toujours disponibles ou à des prix élevés, et de suppléer aux ruptures d'approvisionnement récurrentes. Disposer du droit de circuler et accéder à des réseaux globalisés permet de gérer sa mobilité comme son immobilité, la possibilité de choisir est une marque de différenciation sociale (Van Hear, 2014). 


\section{Le traitement psychiatrique et son articulation à d'autres formes de prise en charge}

26 L'analyse des parcours thérapeutiques montre qu'ils sont, sauf exception, longs, chaotiques et complexes. Lorsqu'une cure psychiatrique est entamée, les médecins demandent à leurs patients de ne pas consommer des produits prescrits par d'autres acteurs afin d'éviter des interférences avec leurs prescriptions. Par contre, ils ne cherchent pas à dissuader leurs patients d'abandonner des pratiques considérées comme « sans danger » (séances de prières, bains mystiques, rituels de protection) tant elles constituent des formes d'habitudes de soin. Ces croyances mystico-religieuses en offrant protection et réassurance aux personnes malades leur évitent de se sentir démunis face au système médical.

27 Quelques patients interrogés dans des services psychiatriques, nous ont conduit à des séances de prières collectives animées par des pasteurs-prêcheurs de nouvelles églises ou des prêtres lors de prières et de cérémonies d'exorcisme. Ces pratiques complètent ce que le suivi psychiatrique n'offre pas ou insuffisamment. D'une part, une forme d'insertion sociale dans des collectifs où les malades ne se sentent pas plus vulnérables ou plus en détresse que l'ensemble des personnes présentes. Un groupe, où ils se définissent " comme les autres, pas fou, normal quoi ". D’autre part, ces séances ritualisées et régulières sont ouvertes et gratuites. Elles revêtent une forme d'accompagnement social qui est décrit comme insuffisant dans les structures médicales qui manquent de ressources. Outre les difficultés financières et logistiques que connaissent les patients et leurs familles, ils expriment régulièrement le sentiment d'être abandonnés à eux-mêmes à l'extérieur des services psychiatriques, ceux-ci se concentrant sur leur fonction première (établir un diagnostic et prescrire des médicaments). Face à une écoute jugée insuffisante et à l'absence d'associations réellement structurées et actives, ils se tournent vers d'autres acteurs qui au contraire mettent en scène leurs capacités à répondre aux attentes sociales des personnes en situation de vulnérabilité.

Un des malades dont nous suivons le parcours depuis 2016 cumule différentes pratiques. Il suit scrupuleusement depuis plusieurs années un traitement psychiatrique à Dalal Xel à Thiès dont il dit ressentir les effets positifs. L'évolution de la posologie et l'espacement des rendez-vous confirment ce jugement. Il conserve néanmoins ses ordonnances en permanence sur lui, car il désire être identifié comme malade si une crise se déclenchait. Parallèlement il se rend régulièrement à l'église catholique Marie reine de l'univers à Thiès où il assiste à des séances d'exorcisme et échange avec le prêtre qui les dirige. Parallèlement, il assiste à des séances de prières à l'Association de Bienfaisance Communautaire - un compromis avec la Communauté qui est une église du réveil.

29 Malgré la reconnaissance qu'il exprime à l'égard de son psychiatre, il explique sa participation à ces séances de prières par le besoin "d'être écouté et soutenu », alors qu'il se heurte à sa famille: " ma mère, elle ne me comprend pas, elle est dure. Je suis critiqué, car je suis en retard. Je n'avance pas dans la vie [a presque 40 ans il est encore célibataire, n'a pas quitté la maison de ses parents et rencontre des difficultés à garder un emploi]. Je ne suis pas comme mes frères, mes sœurs. Si je demande de l'aide, on me dit que je me plains, que je retarde, je coûte ». Face à ces dissensions familiales, tant le slogan que le 
programme de prières sont attractifs pour des malades en quête de reconnaissance et d'insertion sociale [photographie ${ }^{\circ} 2$ ].

Photographie 2 : Programme des séances de prières à l'Association de Bienfaisance Communautaire à Thiès (Petit, 2016)

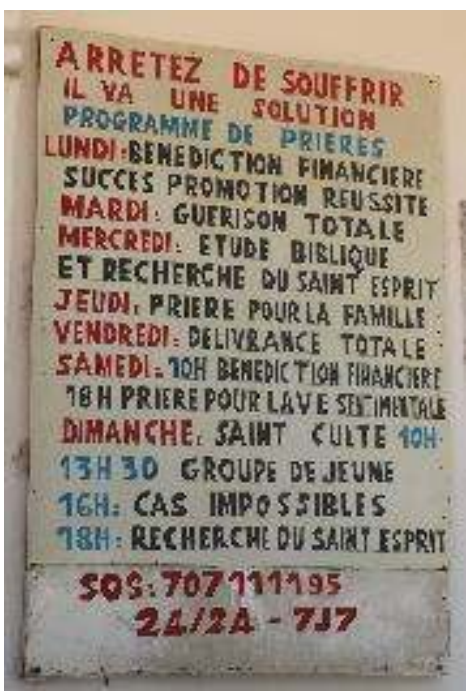

Photo : V. Petit, 2016

\section{La quête thérapeutique, forme d'éducation thérapeutique}

Si le territoire politique qui s'exprime à travers le modèle de santé engendre des inégalités profondes, voire de l'exclusion, les patients se l'approprient grâce à leurs expériences successives et aux compétences qu'ils acquièrent. Loin d'être soumis à l'autorité médicale, les malades-acteurs «rusent avec les structures qui les gouvernent» pour reprendre Michel de Certeau (De Certeau, 1980). Ils préfèrent rechercher une délivrance immédiate et définitive grâce à une intervention mystique, plutôt que d'adhérer sans résistance à un dispositif psychiatrique qui leur semble étranger, difficilement compréhensible et incapable de répondre à leurs attentes. D'autant qu'aux réticences suscitées par l'étiologie médicale s'ajoutent les coûts médicaux et d'accessibilité. Pendant des années le plus souvent, les malades vont aller de marabouts en guérisseurs en églises. Accompagnants et personnes malades sont conduits par la succession de leurs expériences et les discussions avec les psychiatres lorsqu'ils (re) viennent en consultation à renoncer progressivement à l'idée d'une guérison instantanée. Ils sont contraints de faire le deuil de l'idée même de guérir au sens d'une guérison entendue comme un retour à l'état antérieur, c'est-à-dire une situation définie par l'absence de symptômes ou de symptômes devenus insupportables parce qu'ils troublent l'ordre social. Pas plus «qu'il y a un retour à l'innocence physiologique, car il y a irréversibilité de la normativité biologique " (Canguilhem, 2007), il y aurait un retour à une "innocence " psychique. Ces parcours chaotiques constituent une éducation thérapeutique par l'échec en particulier chez des populations ayant un faible niveau de littéracie en santé (Margat et al., 2017). Néanmoins, tous les groupes sociaux font preuve de résistance, à des degrés divers, face à l'idée que la guérison soit une vie avec «la maladie stabilisée » offrant la possibilité d'une réinsertion sociale. 


\section{La médecine prophétique : les centres Roqya}

31 La globalisation de l'offre de soin mystico-religieuse concerne également la promotion de la médecine prophétique ${ }^{7}$. Les centres roqya sont un des acteurs de cette diffusion internationale. Ils ont été cités à plusieurs reprises par des malades ou des accompagnants comme un des recours qui avaient été expérimentés ou qui étaient envisagés comme alternative ou complément au traitement psychiatrique. Compte tenu des croyances populaires selon lesquelles la folie ne se guérit jamais réellement en dépit de phases de rémission et de l'étiologie qui l'associe à la possession, les familles recherchent une solution où la cause du mal, nécessairement extérieure à l'individu, pourrait être extirpée définitivement.

\section{Un positionnement paradoxal}

Alors que la médecine prophétique s'ancre dans l'islam et se réfère directement au coran, le positionnement des centres roqya permettent d'intéresser à ce type de soins une large partie de la population. En effet, ceux-ci annoncent soigner « les blocages dans la vie, les états mentaux et de santé anormaux, les possessions des djinns, le mauvais ceil (thiat) " [photographies 3,4 et 5]. Il s'agit d'extraire, grâce à des captages, des procédés purgatifs et des incantations, les djinns, les shayatins (les diables), les maux liés à l'ensorcellement et à la possession, et de détruire les autels khamb dans les demeures. Les multiples références mobilisées dans leur présentation (islam, culte des ancêtres, psychologie, psychiatrie, médecine) permettent à chacune des composantes sociales d'y trouver un discours compatible avec ses aspirations. Cette approche que l'on pourrait qualifier d'œcuménique dans le sens où elle produit un discours audible par tous, se retrouve également chez les prêtres-exorcistes et les pasteurs. Ils délivrent tous « $d u$ mal » et « la souffrance n'a pas de religion».

Photographies 3, 4 et 5 : Banderole du centre roqya à Ziguinchor, affichette à l'intérieur de la salle d'attente, livre exposé sur le bureau du directeur (Petit, 2018)

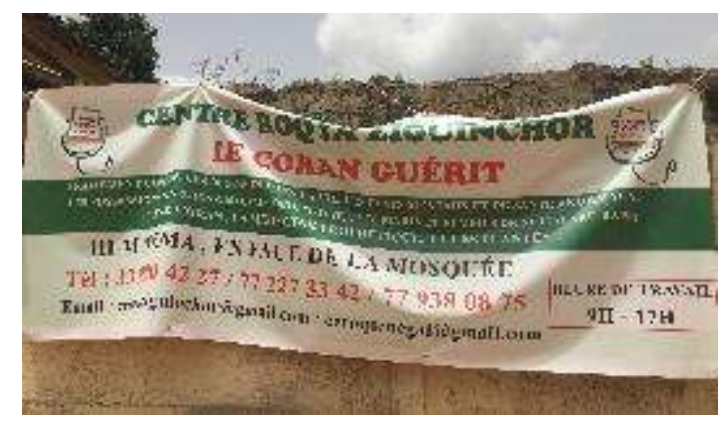



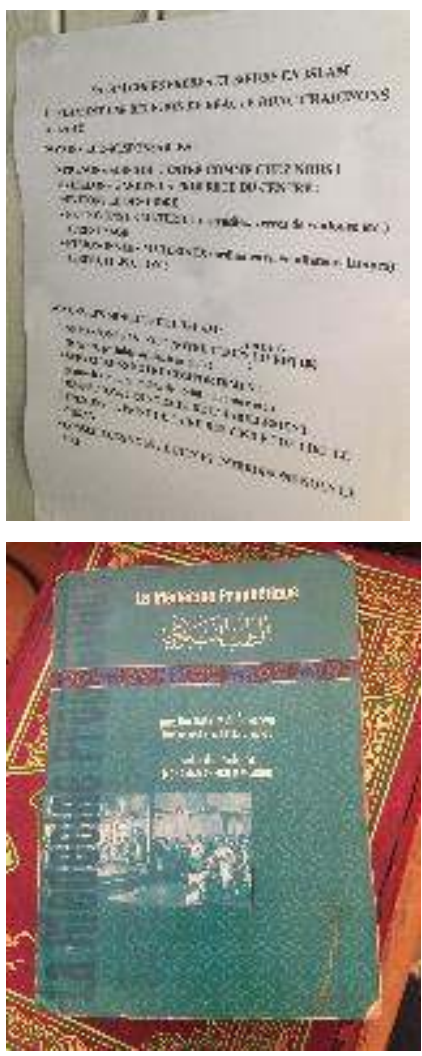

Photos: V. Petit, 2018

33 Néanmoins dans un marché thérapeutique concurrentiel, les tenants du «Coran qui guérit » opposent la pureté de leurs pratiques en raison de leur ancienneté et de leur authenticité à celles des marabouts, en particulier au niveau local. Ils s'attribuent une légitimité extérieure incontestable en remontant aux sources arabes de l'islam. Le centre roqya a donc pour vocation de répondre « aux pratiques religieuses, à ceux qui ont pêché dans le besoin de se soigner. (...) On interdit les sacrifices, on peut tuer un boeuf un mouton pour partager, mais ça n'est pas pour un sacrifice pour les démons, pour un pacte avec les ancêtres. Quand tes parents ont fait des pactes de protection de la famille, ces pratiques rituelles ne sont pas islamiques. Si tu deviens musulman tu romps le pacte, les démons agissent ». Inversement les pratiques des marabouts locaux auraient été perverties par leur trop grande proximité avec les croyances et les coutumes locales. Ceux qu'ils désignent comme " les talebs ou les voyants au Sénégal » ne seraient que de pâles imitations de la médecine prophétique (" Allah! Le Sénégal copie beaucoup et copie mal »). La biomédecine n'échappe pas non plus à la critique :

«L'Europe, c'est pas l'Afrique ! Il y a des spécificités selon les réalités, mais les gens font des amalgames, ils copient mal, il y a des maladies, des problèmes. »

\section{Un réseau international}

Le centre Roqya du quartier HLM néma à Ziguinchor dans lequel nous avons enquêté est ainsi inclus dans le réseau des centres roqya qui se multiplient ces dernières années au Sénégal (Dakar, Kaolack, Richard Toll, Mboro, Mbour, Ziguinchor). Cette diffusion touche également la Côte d'Ivoire, le Bénin, la Guinée, le Mali, la France et l'Angleterre. Un des responsables du centre formé au management-marketing précise qu'être intégré dans ce style de réseau " permet aux membres d'aller de l'un à l'autre, d'être formés, 
d'harmoniser le travail, d'unifier, c'est mieux pour la visibilité, la propagande ». Parallèlement à la circulation des responsables de centres et des " capteurs ", l'appartenance au réseau favorise l'approvisionnement en produits et en médicaments. Des références récurrentes à l'Arabie saoudite et à des États arabo-musulmans ponctuent les discours et marquent l'inscription originelle des pratiques. Les patients peuvent par exemple acheter de l'eau bénite en provenance de la Mecque. D'autres produits sont proposés afin de répondre à des préoccupations plus modernes telles la perte de poids, le contrôle du stress ou de l'anxiété. Il s'agit par exemple de compléments alimentaires, de vitamines pour améliorer sa forme et son apparence physique, et des traitements contre le diabète. Ces " médicaments de partout dans le monde " arrivent par bateaux à Dakar et Kaolack en provenance d'Asie (Corée, Malaisie, Thaïlande, Arabie Saoudite), d'Europe, du Maghreb. Remplaçant les traditionnelles ventouses en verre, le matériel technique pour effectuer « les captages » provient de Corée [photographies 6, 7 et 8]. Il s'agit à travers les sucions provoquées par les ventouses en caoutchouc d'extirper les djinns qui sont à l'origine des " blocages » dans la vie et des problèmes de santé des personnes qui viennent consulter. Par « blocages ", les acteurs sous-entendent qu'ils ne peuvent " avancer » dans la vie ou qu'ils voient leurs projets matrimoniaux, familiaux, professionnels ou migratoires contrecarrer par des actions de sorcellerie. Ces situations ont également des répercussions sur leur état de santé.

Photographies 6,7 et 8 : La boîte à ventouses en provenance de Corée pour les " captages ", des médicaments en provenance de Malaisie (Petit, 2018)
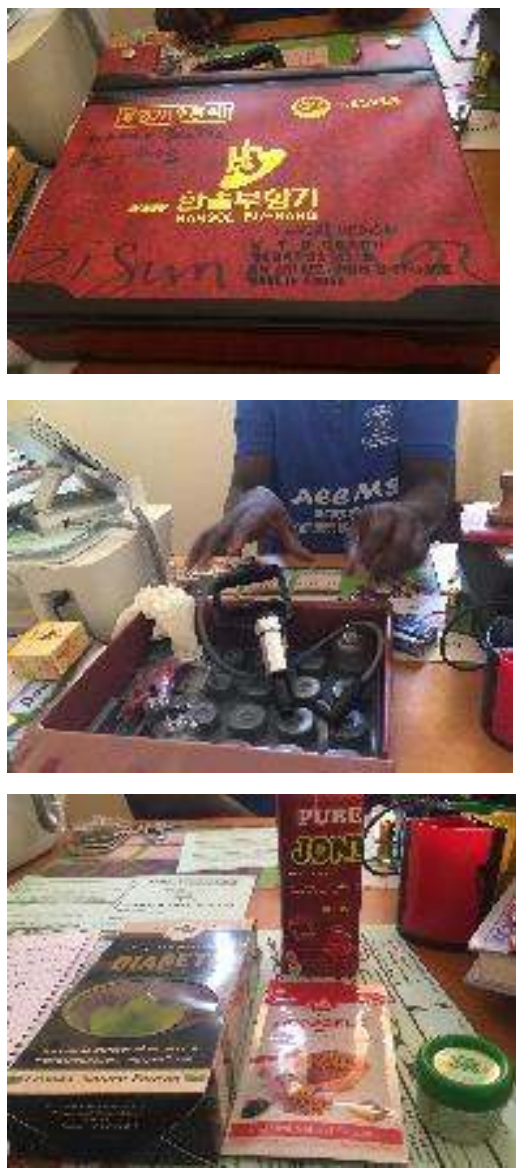

Photos : V. Petit, 2018

Revue francophone sur la santé et les territoires, Les circulations en santé : des produits, des savoirs, des personnes en mouvement 


\section{Charia de la santé et psychothérapie} s'inscrit totalement et uniquement dans le domaine de la religion, elle n'entre donc pas en concurrence, ni avec les structures hospitalières de Ziguinchor, ni avec les tradipraticiens, quels qu'ils soient. Insistant sur l'ancrage dans l'islam, il précise : « Nous travaillons selon le principe de la charia de la santé. Le coran guérit car il répond à tous les problèmes (...) La base c'est le coran, c'est impératif. Ici quand on a le temps on fait du renforcement au niveau de la mémorisation du coran ». Les personnes qui entament un traitement doivent se plier à des règles de vie afin de prendre soin de leur état physique et mental: "Éviter de se mettre en colère, car ce n'est pas bon pour un état psychologique stable, ne pas approcher de ce qui peut te détruire ton esprit: la drogue, c'est comme quand tu es fou, quand tu es ivre, ne médisez pas ». Cette charia de la santé interdit la consommation de drogue et d'alcool, le suicide, la dépigmentation de la peau. Elle prescrit certaines attitudes insistant sur la dimension communautaire de la santé : « Tous les problèmes sociaux, la vie quotidienne, le coran n'a laissé en rade aucun aspect. En tant que musulman on oriente son comportement: avoir une bonne relation avec son seigneur, il a un cœeur avec de la pitié, amour, il ne peut pas faire de mal; avoir des relations avec sa famille: faire le bien à son prochain; la famille africaine c'est une famille de solidarités. Avoir de bonnes relations avec son physique avec soi-même. Si tu ne vis pas ces liens, tu vas avoir des problèmes, vivre dans la souffrance, tu ne vas pas te concentrer dans ton travail, tes études. Notre santé est dépendante de notre positionnement social et céleste, de la qualité de notre rapport aux autres et à soi ». Les personnes suivies reçoivent une fiche rédigée en français et en arabe leur rappelant les prières de protection, les incantations à réciter et les rituels à accomplir quotidiennement.

Après avoir insisté sur les dimensions morale et communautaire de la santé, le responsable du centre tient un discours plus psychologisant et centré sur l'individu (« Il faut prendre soin de soi, se respecter, avoir de l'estime de toi-même "). Lorsqu'un malade se présente au centre, il est reçu par le directeur qui conduit un entretien " pour identifier le problème uniquement grâce à des questions, il n'y a pas d'examens [sous-entendu clinique] ». À côté du bureau dédié aux consultations, une salle est consacrée à la psychothérapie alors que personne n'est formé à la psychologie dans le personnel du centre. Selon le directeur, les malades doivent être accompagnés " au-delà de la cure avec captage et médicaments par la psychothérapie ». Lorsqu'ils sont face à des malades " agités ou nerveux ", les thérapeutes qui se désignent comme étant des " capteurs » du centre roqya leur donnent des comprimés de Vogalène ${ }^{\circledast}$ (médicament antiémétique utilisé en cas de nausées et de vomissements) dont ils utilisent les effets secondaires. Ils achètent ce médicament à la pharmacie du quartier et sans prescription médicale. Ils le désignent comme un somnifère. Alors que l'accueil des malades, leur enregistrement, les pratiques thérapeutiques et les produits commercialisés répondent à des protocoles formalisés et standardisés réalisés à l'intérieur d'espaces dédiés à chaque opération (entretien, captage, prières, psychothérapie), le discours qui les accompagne suggère que le suivi est individualisé et adapté aux troubles somatiques et psychiques de chaque malade. Cette valorisation de l'individu, même si elle est constamment articulée aux liens sociaux, s'oppose clairement à la " société de la honte " qu'évoquent psychiatres et psychologues en décrivant les mécanismes de répression et d'intériorisation à l'œuvre dans la société sénégalaise. 


\section{Les « villages thérapeutiques » des marabouts}

\section{Se légitimer en s'inscrivant dans l'héritage psychiatrique} mettre en œuvre au Sénégal. Les origines et la circulation de cette expérimentation ont été retracées par le psycho-sociologue René Collignon. Il existait en Europe des pratiques philanthropiques anciennes comme celles des Quakers en Angleterre, des hébergements familiaux et des accueils villageois dont le plus connu est celui de Geel en Belgique (Collignon 1983, 2001). Ces expériences de prise en charge ont interpellé les psychiatres européens, avant d'être testées en Afrique par des psychiatres formés dans les États colonisateurs. Le modèle de Geel a servi d'inspiration au psychiatre Thomas A. Lambo au Nigéria lorsqu'il établit en 1954 le « Aro village program ». Il met en place un accueil dans des villages yoruba en accord avec les chefs et en collaboration avec les guérisseurs locaux: " le contact avec un environnement social normal constitue l'aspect le plus important de son programme thérapeutique. Ce milieu, plus en harmonie avec le niveau de fonctionnement altéré du patient que ce que pourrait lui proposer la structure artificielle de l'hôpital par la cohabitation avec des gens présentant un meilleur équilibre émotionnel que lui dans un groupe tolérant, ménage au patient un espace social où il peut s'engager dans une confrontation avec la réalité par la participation progressive aux activités communes sans peur de nouveaux traumatismes » (Collignon, $1983:$ 290-291). À partir de 1969, Collomb et son équipe cherchent à établir des villages thérapeutiques, la réalisation qui correspond le mieux au projet initial est celle du village de Kénia à quelques kilomètres de Ziguinchor. Le village ouvre en 1974, mais à terme le projet n'est pas viable faute d'un engagement suffisant de l'institution psychiatrique et en raison des difficultés concrètes de mise en oeuvre.

Presque cinquante ans plus tard, les villages thérapeutiques et à travers eux la psychiatrie communautaire, n'apparaissent plus comme une solution soutenue par les psychiatres sénégalais, alors qu'elle est revendiquée par certains marabouts qui s'inscrivent dans l'héritage de la psychiatrie culturelle. C'est le cas deux marabouts à Mawa et Tobor des villages non loin de Ziguinchor. Les marabouts rappellent que H. Collomb a travaillé avec des guérisseurs, voir même directement avec leur père, lorsqu'il a mis en place le village de Kénia ce qui les légitime à poursuivre son œuvre ; et ce, d'autant plus qu'elle est abandonnée par la psychiatrie sénégalaise et que les psychiatres sont très réticents à collaborer avec eux. C'est ainsi que Serigne Dame Dieng à Tobor a construit plusieurs cases d'un confort sommaire où il accueille avec ses disciples des personnes malades qui sont laissées à sa garde par des familles usées par des années de quête thérapeutique ou qu'il recueille dans les rues des villes de la région. Nous avons dénombré une quarantaine de malades. Ils sont principalement originaires de Casamance, mais certains sont d'anciens migrants revenus d'Europe, d'autres proviennent des pays frontaliers voisins (Guinée Conakry, Guinée Bissau, Gambie) (Petit, 2019).

\section{La doctrine mouride actualisée}

Le marabout récupère à la fois l'expression "village thérapeutique " initiée par les psychiatres et l'idée selon laquelle il faut replacer le malade dans un cadre social qui

Revue francophone sur la santé et les territoires, Les circulations en santé : des produits, des savoirs, des personnes en mouvement 
soit favorable à sa guérison. Il s'inscrit dans un processus pluriel de légitimation qui repose à la fois sur sa propre notoriété qu'il utilise et renforce en mêlant actions sociale (création de l'Association pour la réinsertion sociale des déficients mentaux et des enfants en situation difficile) et thérapeutique, en s'appuyant sur la réputation du centre de santé mentale Emile Badiane avec lequel il souhaite développer une collaboration et enfin celle de biomédecine dont il manipule certains médicaments en complément à ses remèdes (Egrot, 2015).

40 Le village était jugé sain et propice à une récupération, à l'inverse du milieu urbain considéré comme pathogène par les médecins. Le malade est placé sous le contrôle d'une communauté qui l'accepte, il est dans son cadre socioculturel et il participe aux activités quotidiennes. La perspective initiale est retranscrite dans la doctrine mouride du travail ${ }^{9}$ : c'est grâce à une discipline physique, morale et spirituelle, au travail quotidien dans les champs d'arachide, au respect des règles de la communauté et des rites prescrits que le psychisme ira mieux (fumigations, plantes et racines collectées dans la forêt, bains mystiques, etc.). Néanmoins certains malades sont entravés " afin d'éviter les fugues et qu'ils se perdent en quittant la concession ». Les menottes et les chaînes ne sont nullement dissimulées [photographie 11], elles sont présentées comme une mesure de protection du malade face à une société qui maltraite ses malades. Leur usage est légitimé en référence à des daaras ${ }^{10}$ où on enchaîne les enfants voulant fuir leur condition.

41 Lorsque les malades arrivent, ils sont inscrits dans un registre et une fiche d'entrée est établie à leur nom [photographie 9]. Le marabout, comme les responsables des centres Roqya, a réalisé des documents en s'inspirant de ceux des centres psychiatriques; il extrait des informations tirées des documents médicaux (ordonnances, compte rendu d'examens, carnet de suivi). Sa nosologie est un bricolage reprenant les grandes classifications internationales qu'il agrémente d'appellations de son invention ou trouvées sur internet. En consultant ses registres et les fiches, on constate qu'ils ne sont ni actualisés ni soigneusement complétés. Ayant créé une association, ce marabout est tenu par des obligations légales. La gestion "administrative» des malades est présentée comme un gage de professionnalisme, "son village thérapeutique » ayant pour objet " la réinsertion des malades ». Les encadrants, c'est-à-dire les disciples du marabout, insistent sur l'accompagnement quotidien des malades dans les soins, les activités collectives, ils réalisent également des tests "psychologiques» de leur invention afin d'évaluer les activités que les malades peuvent réaliser ("il faut éveiller le malade »). Le marabout et ses disciples conservent le carnet de suivi médical et les ordonnances des malades [photographie 10]. Ils utilisent ces dernières afin d'acheter des médicaments à des prix négociés au dépôt central à Ziguinchor où s'approvisionnent les pharmacies de la région.

42 Les talibés utilisent sous forme d'automédication des médicaments tels que l'artane ${ }^{\circledast}$ ${ }^{11}$, la rispéridone ${ }^{12}$, le largactil ${ }^{\circledR}$, le lexomil ${ }^{\oplus 13}$, des somnifères, lorsque les traitements dits mystiques (bains, prières, fumigations) ou le travail aux champs ne suffisent pas à calmer les malades qui leur ont été confiés ou qu'ils ont recueilli dans la rue. Selon qu'il faille stimuler ("éveiller») ou au contraire calmer la personne, dans certaines situations des médicaments sont détournés de leurs usages initiaux à des fins psychotiques ou en raison des effets secondaires qui ont été observés. Ces usages entrent parfois en interaction avec la consommation de cannabis ou d'autres stupéfiants dont la consommation s'est répandue dans la population sénégalaise en 
particulier chez les jeunes ${ }^{14}$. Différentes études ont montré dans le cas de la capitale malienne que la prescription, la délivrance et la consommation de psychotropes et neuroleptiques constituaient un problème de santé publique, et ce en dépit des progrès observés, compte tenu de la relative facilité avec laquelle les individus obtenaient dans les officines pharmaceutiques les produits nécessaires à leur automédication sur simple demande orale, en présentant de vieilles boîtes ou d'anciennes ordonnances. Ils ont montré que le lexomil ${ }^{\oplus}$, était le médicament le plus prescrit et le plus demandé, suivi par le tranxène ${ }^{\oplus}$, le témesta ${ }^{\oplus}$, le valium ${ }^{\circledast}$ et le myolastan $^{\circledast}$ (Fane, 2010 ; Singaré, 2006 ; Kanadji, 2004). Ces travaux montrent, outre les manquements dans la rédaction des ordonnances (absence du nom du prescripteur, du nom du patient, de la date, de la durée, de la posologie), que les prescripteurs ne sont pas en majorité des spécialistes (psychiatres ou neurologues), mais des généralistes du secteur privé, des infirmiers, des sages-femmes. Ces dernières catégories ne maitrisent en raison d'une formation insuffisante en psychiatrie pas le bon usage de ces médicaments comme cela a été montré dans le cas de Dakar (Dièye et al., 2007).

Ces formes d'automédications apparaissent dans des récits d'accompagnants. Ils témoignent de la présence dans certaines pharmacies familiales de neuroleptiques et d'anxiolytiques. Vendus par des pharmaciens ou ramenés par des migrants, ces médicaments ne sont plus associés à une pathologie et à un malade spécifique. Indépendamment d'une prescription médicale, ils sont identifiés comme offrant une solution ponctuelle de relatif contrôle d'un état de violence ou de désinhibition évitant ainsi de se rendre dans un service psychiatrique et d'être confronté à des coûts sociaux et financiers insurmontables.

Photographies 9, 10, 11 : Le registre de l'Association pour la réinsertion sociale des déficients mentaux et des enfants en situation difficile, le carnet de suivi du centre psychiatrique, la pose des entraves à une personne malade (Petit 2017 et 2018)

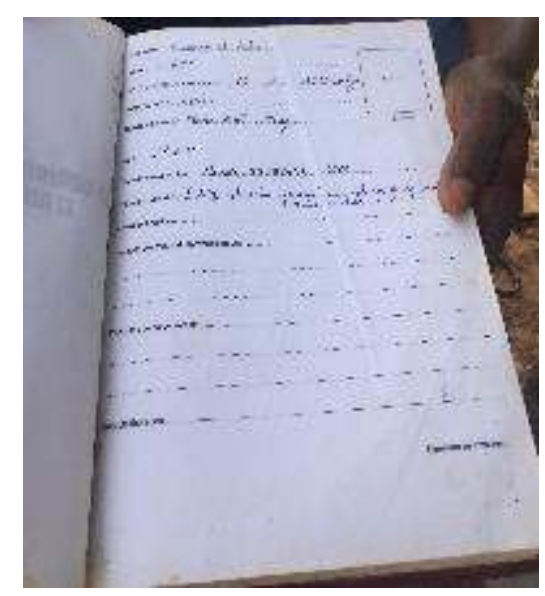




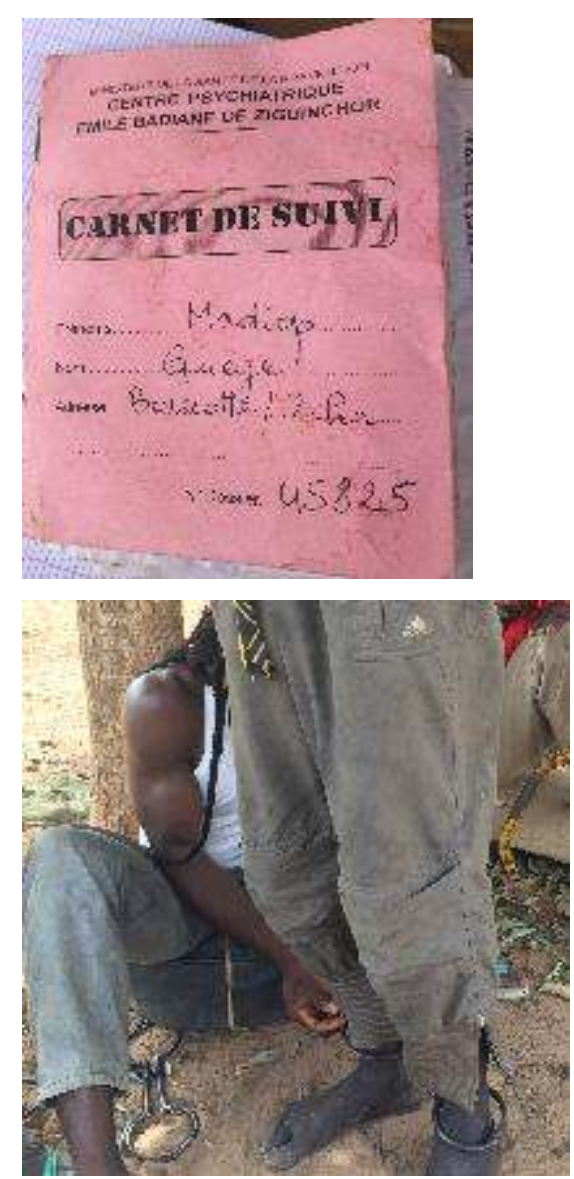

Photos : V. Petit, 2017 \& 2018

\section{Conclusion}

\section{Les circulations d'une société en souffrance}

44 L'ensemble des acteurs convergent vers l'idée que la société sénégalaise est « en crise, en souffrance " et que les individus expriment des troubles psychiques qui traduisent leurs difficultés face à un environnement qu'ils ne parviennent plus à maîtriser alors que les institutions attendent d'eux qu'ils s'ajustent à de nouvelles injonctions et qu'ils soient performants. Un psychiatre observe qu' "Il y a un coming out de plein de choses. Les gens, ils expriment mieux ou d'une autre façon leur mal-être social. Et on voit beaucoup plus maintenant, de façon plus récurrente, des pathologies comme la dépression; là, c'est la dépression qu'on retrouve dans la littérature (...) parce que, tout simplement, les valeurs ont bougé » (LB, psychiatre, 2016). Ce constat va dans le sens de Laure Murat qui affirme que le délire est sans doute la chose la mieux partagée et qu'il est l'« investissement inconscient des individus aux prises avec les guerres, les massacres, la violence, l'horreur économique » (Murat, 2011). De leurs côtés, Gilles Deleuze et Félix Guattari définissaient le délire comme " l'investissement inconscient d'un champ social historique " (Deleuze et Guattari, 1972). Si la volonté de changer la société et de moderniser le pays est exprimée par les différents gouvernements successifs, ces mutations ne sont pas suffisamment socialement et politiquement accompagnées. D'une part, l'instauration de la couverture médicale universelle est loin d'être généralisée. D’autre part, elle ne 
modifie pas l'offre de soin en santé mentale qui demeure insuffisante et inégalement répartie sur le territoire, le dernier rapport de la Direction de la Santé Mentale souligne le décalage entre les moyens dont elle dispose et les objectifs à atteindre afin d'assurer une meilleure santé mentale aux groupes identifiés comme les plus fragiles (Tine, 2019). Le premier défi à relever pour cette institution est celui qui consiste à mobiliser des partenaires afin de collecter les fonds nécessaires à la concrétisation des ambitions énoncées.

\section{Des circulations comme évitement ou alternatives à la psychiatrie}

Nous l'avons vu les personnes malades et leurs parents confrontées à un problème de comportement psychique recherchent toutes les solutions thérapeutiques qui permettent de retarder le recours à la psychiatrie compte tenu des coûts élevés que celle-ci implique et de la construction de leur étiologie personnelle. Ces circulations sont régies également par le ressort du secret en réponse à la stigmatisation en dépit de la médiatisation croissante des questionnements «psy » autour de la dépression et du suicide par exemple. Les personnes malades sont amenées à entreprendre des circulations plurielles afin d'expérimenter des solutions alternatives en mobilisant les ressources de leurs territoires et leurs réseaux à différentes échelles, en particulier locale et régionale. Ces circulations d'acteurs (personnes malades, thérapeutes) croisent les circulations de produits et de médicaments qui sont ancrées dans des échelles plus globales et impliquent parfois les migrants comme passeurs. Les inégalités sociales s'expriment à travers les compétences et les capitaux qui permettent de choisir les types de productions et les modes de circulations. Les groupes moins bien dotés et les plus vulnérables faute d'accéder à certaines circulations et d'entrer un parcours thérapeutique qui se structure progressivement autour d'une cure psychiatrique se retrouvent dans l'errance également en raison d'ancrages familiaux et sociaux suffisamment actifs.

\section{Vers une psychologisation de la société ?}

Dans un contexte de concurrence thérapeutique et d'individuation accrue, la rhétorique de l'épanouissement personnel trouve un écho indéniable dans la société sénégalaise et fait l'objet de diverses déclinaisons mystico-religieuses. Ce sont surtout les marabouts, les guérisseurs, les pasteurs, les prêtres exorcistes qui s'emparent des injonctions internationales quant au bien être individuel et collectif. Ils agglomèrent un ensemble de symboles, d'imaginaires, de concepts issus d'univers aux légitimités antinomiques (religions, traditions, biomédecine) afin d'élaborer un ensemble de discours et de pratiques susceptibles de combler le vide laissé par les institutions politiques et médicales. Ils laissent espérer aux personnes malades et à leur famille l'espoir d'une guérison définitive, alors que les médecins, comme dans le cas des maladies chroniques, sont contraints d'annoncer à leurs patients des traitements longs et onéreux.

47 Les circulations idéelles induisent des questionnements quant à l'appropriation de savoirs, de pratiques et de produits, car ils sont manipulés par des profanes. Les précautions techniques et éthiques se dissolvent au fil de diffusions sauvages et non régulées par l'Etat à travers la reconnaissance des spécificités professionnelles. Le 
développement de la psychologie avec la création d'une association des psychologues du Sénégal, regroupant des psychologues sénégalais et français formés en Europe traduit le besoin de sortir des codes moraux et sociétaux afin d'affirmer cette quête individuelle en «psy» [photographie 12]. La demande d'un accompagnement psychologique peut être rapprochée des processus anthropologiques conduisant des individus à "sortir du groupe " afin d'échapper à leurs conditions d'origine et en assumant les décisions relatives à leur destinée individuelle. Cette prise de distance avec les institutions traditionnelles s'effectue de manière négociée et dans un contexte de «modernité insécurisée » telle que la définit Pierre Joseph Laurent, si elle semble hautement désirable elle n'est pas exempte de souffrances psychiques compte tenu des adaptations à la modernité qu'elle implique dans un contexte marqué par l'absence relative de l'Etat (Laurent, 2007). On peut s'interroger sur l'effacement et la résistance des institutions politiques et médicales dans une certaine mesure face à la psychologie, cette discipline n'étant pas enseignée au Sénégal, compte tenu du destin de la psychiatrie. Cette dernière associée aux idées de contrôle social et d'adaptation répond aux souhaits des institutions sociales et politiques, tandis que la psychologie et la psychanalyse en travaillant à l'émancipation des individualités portent un projet plus subversif dans « l'Afrique des individus ».

Photographie 12 : Peinture sur verre représentant le psychologue, nouvelle profession représentée dans l'art populaire (Petit, Dakar 2017)

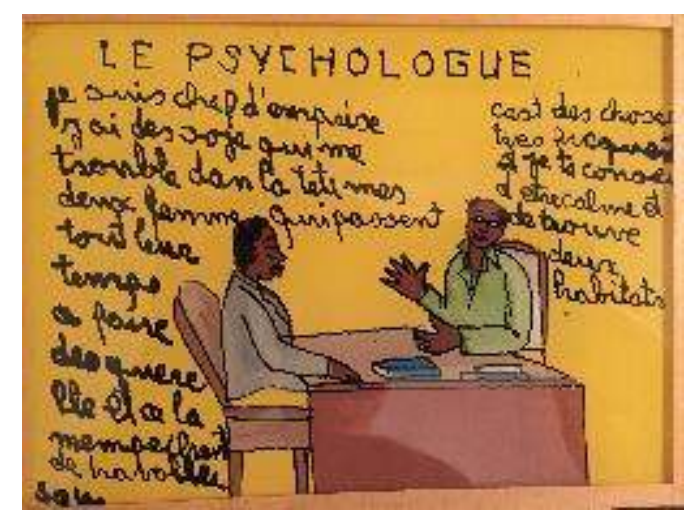

Photos : V. Petit, Dakar 2017

\section{BIBLIOGRAPHIE}

Akyeampong E., Hill A. G., Kleinman A., 2015, The culture of mental illness and psychiatric practice in

Africa, Indiana University Press, Bloomington and Indianapolis, $349 \mathrm{p}$.

Ansd D. Des S.D. Et S., 2018, « La population du Sénégal en 2017 », Dakar, Ministères de

l'Economie, des finances et du plan -Agence Nationale de la Statistique et de la Démographie.

Atlani-Duault L., Vidal L., 2013, « Le moment de la santé globale. Formes, figures et agendas d'un miroir de l'aide internationale », Revue Tiers Monde , 215 , 3, p. 7-16.

Revue francophone sur la santé et les territoires, Les circulations en santé : des produits, des savoirs, des personnes en mouvement 
Becker C., Collignon R., 1999, «Épidémies et médecine coloniale en Afrique de l'Ouest », Cahiers d'études et de recherches francophones / Santé , 8 , 6, p. 411-416.

Canguilhem G., 2007, Le normal et le pathologique, PUF, Paris.

Collignon R., 1983, « A propos de psychiatrie communautaire en Afrique noire. les dispositifs villageois d'assistance ", Psychopathologie africaine , X IX , 3, p. 287-328.

Collignon R., 1984, « La lutte des pouvoirs publics contre les "encombrements humains" à Dakar ", Canadian Journal of African Studies / Revue canadienne des études africaines , 18 , 3, p. 573-582.

Collignon R., 1999, «Le traitement de la folie au Sénégal à l'époque coloniale », dans Enfermement, prisons et châtiments en Afrique du $19^{e}$ siècle à nos jours, Karthala, Paris, p. 227-258.

Collignon R., 2002, « Pour une histoire de la psychiatrie coloniale française : à partir de l'exemple du Sénégal », L'Autre , 3 , 3, p. 455-480.

De Certeau M., 1980, L'invention du quotidien tome 1 : arts de faire , Paris, Union Générale d'Editions. Deleuze G., Guattari F., 1972, L'Anti-CEdipe. Capitalisme et schizophrénie I ., Paris, Editions de Minuit. Di Méo G., 2006, « Les territoires de l'action », Bulletin de la Société Géographique de Liège, Société Géographique de Liège , p. 7-17.

Diagne P.M., Lovell A., 2019, « De l'accompagnant familial au " mercenaire » : les travailleurs subalternes et la transformation de la psychiatrie publique au Sénégal », Sciences sociales et santé , 37,2 , p. 75-99.

Dièye A.M., Ndiaye Sy A., Yoro Sy G., Aw Diallo A., Diarra M., Ndiaye M., Faye B., 2007, «Prescription des benzodiazépines par les médecins généralistes du privé à Dakar : enquête sur les connaissances et les attitudes ", Thérapie , 62 , 2, p. 163-168.

DilgerH., Kane A., Langwick S.A., 2012, Medecine, mobility, and power un global Africa. transnational health and healing, Indiana University Press, Bloomington and Indianapolis.

Diop M.C., 1997, « L'administration sénégalaise et la gestion des “fléaux sociaux”. L'héritage colonial. », dans Becker C., M baye S., Thioub I. (dirs.), AOF : réalités et héritages, sociétés ouestafricaines et ordre colonial 1895-1960 ., Direction des archives du Sénégal, Dakar, Sénégal, p. 1137-1158.

Diop M.C., 2010, Le Sénégal des migrations. Mobilités, identités et sociétés , Paris.

Eaton Julian, Mccay L., Semrau M., Chatterjee S., Baingana F., Araya R., Ntulo C., Thornicroft G., Saxena S., 2011, « Scale up of services for mental health in low-income and middle-income countries ", The Lancet , 378, p. 1592-603.

Egrot M., 2015, « Produits frontières, légitimité, confiance et automédication : interférences autour de quelques médicaments néotraditionnels circulant en Afrique de l'Ouest », dans Baxerres C., Guienne V., Houngnihin R., Marquis C. (dirs.), Actes des rencontres Nord/Sud de l'automédication et de ses déterminants , IRD Université de Nantes Université d'Abomey Calavi, Cotonou, p. 166-184.

Fane S., 2010, Etude des bonnes pratiques de dispensation es psychotropes et neuroleptiques dans les officines du district de Bamako, Thèse de doctorat, Bamako, Université de Bamako, Faculté de médecine, phamarcie et d'odon-stomatologie, $78 \mathrm{p}$.

Funk M., Benradia I., Roelandt J.-L., 2014, « Santé mentale et soins de santé primaires : une perspective globale », L'information psychiatrique , 90 , 5, p. 331- 339.

Garrett L., 2007, « The Challenge of Global Health », Foreign Affairs , 86 , 1, p. 14-38. 
Global Burden of Disease Study 2013 Collaborators , 2015, « Global, regional, and national incidence, prevalence, and years lived with disability for 301 acute and chronic diseases and injuries in 188 countries, 1990-2013: a systematic analysis for the Global Burden of Disease Study $2013 »$, Lancet , 386 , p. 743-800.

Gureje O., Alem A., 2000, « Elaboration des politiques de santé mentale en Afrique », Bulletin of the World Health Organization , 78 , 4, p. 475-482.

Hane F., 2017, « Production des statistiques sanitaires au Sénégal : entre enjeux politiques et jeux d'acteurs ", Santé publique , 29 , 6, p. 879-886.

Kanadji M., 2004, Etude de la prescription et de la consommation des anxiolytiques dans le district de Bamako, Thèse de doctorat, Bamako, Université de Bamako, Faculté de médecine, phamarcie et d'odon-stomatologie, $81 \mathrm{p}$.

Kane A., 2001, « Diaspora villageoise et développement local en Afrique : le cas de Thilogne association développement -Persée ", Hommes et migrations , 1229 numéro thématique : Vie associative, action citoyenne, p. 96-107.

Kastler F., 2011, « La santé mentale en Afrique : un défi oublié ou u ne réponse institutionnelle inadaptée ? », dans Santé internationale , Presses de Sciences Po P FNSP , Paris, p. 169-177.

Kleinman A.G., Lockwood E., Usmani S., Chisholm D., Marquez P.V., Evans T.G., Saxena S., 2016, « Time for mental Health to come out the shadows », The Lancet , 387 , 4, p. 2274-2275.

Koundoul A., 2015, « La professionnalisation de l'accompagnement des malades en milieu psychiatrique au Sénégal », Le journal des psychologues , 10 , 332, p. 42-51.

Laurent P.-J., 2007, « L'offre de guérison des Assemblées de Dieu du Burkina Faso. Un bricolage en train de se faire entre la société mythique et la modernité globalisée. ", Ethnographiques.org ,14 .

Lessault D., Robin N., Georg O., 2015, « Sénégal », dans Simon G. (dir.), Dictionnaire des migrations internationales. Approche géohistorique, Paris, Colin, p. 471-475.

Ly M., Petit V., Pizzolato G., 2014, « La migration internationale face à la santé mentale au Sénégal : récits, discours et imaginaires ", dans Canut C., Mazauric C. (dirs.), Les mots de la migration. Mise en récits et en images des migrations transafricaines, Paris, Le cavalier bleu, p. 221-239.

Margat A., Gagnayre R., Lombrail P., De Andrade V., Azogui-Levy S., 2017, « Interventions en littératie en santé et éducation thérapeutique : une revue de la littérature ", Santé publique , 29,6 , p. 811-820.

Mnookin S., 2016, « Out of the shadows. Making Mental Health a Global Development Priority », World Bank \& World Health Organization.

Murat L., 2011, L'homme qui se prenait pour Napoléon. Pour une histoire politique de la folie , Gallimard, Paris.

Niang Diene A., 2019, La gouvernance de la santé. Enjeux et pratiques au Sénégal , Paris, L'Harmattan.

Patel V., Araya R., Chatterjee S., Cohen A., De Silva M., Hosman C., Mcguire H., Rojas G., 2007, " Treatment and prevention of mental disorders in low-incomes and middle-income countries", The Lancet , 370 , p. 991-1005.

Patel V., Saxena S., Lund C., Thornicroft G., Baingana F., Bolton P., 2018, « The Lancet Commission on global mental health and sustainable development ", The Lancet, october 9, p. 46.

Petit V., 2013, Counting population. Understanding societies. Toward an interpretative demography , Dordreich, Springer (Demographic transformation and socio-economic development). 
Petit V., 2019, « Retours contraints de migrants internationaux au Sénégal : dilemmes familiaux face à la maladie mentale », Revue européenne des migrations internationales , 34 , 2-3, p. 131-158.

Petit V., Charbit Y., Kreager P., Qureshi K., 2020 (à paraître), Anthropological Demography of Health , Oxford University Press, Oxford.

Petit V., Wang S., 2019, « Editorial. Santé mentale en migrations internationales », Revue européenne des migrations internationales , $34,2 / 3$, p. 7-20.

Prince M., Patel V., Saxena S., Maselko J., Maj M., Phillips M.R., Rahman A., 2007, « No health without mental health », The Lancet , 370 , p. 859-877.

Saxena S., Thornicroft G., Knapp M., Whiteford H.A., 2007, « Resources for mental health: scarcity, inequaty, and inefficency », The Lancet , 370 , September 8, p. 878-889.

Singaré S.D., 2006, Dispensation des psychotropes dans le milieu urbain, cas de Bamako, Thèse de doctorat, Bamako, $106 \mathrm{p}$.

Storper-Perez D., 1974, La folie colonisée , Paris, Maspéro (Textes à l'appui/Psychiatrie), 156 p.

Strauss A., Fegerhaugh S., Suczek B., Wiener C., 1985, « Maladie et trajectoires ", dans Anselm Strauss. La trame de la négociation. Sociologie qualitative et interactionnisme, L'Harmattan, Paris (Logiques sociales), p. 191-240.

Tine J.A., 2019, « Rapport d'activité 2018 de la Division de la Santé Mentale et perspectives 2019-2020».

Van Hear N., 2014, « Reconsidering Migration and Class », International Migration Review , 48 , p. S100-S121.

Vigo D., Thornicroft G., A tun R., 2016, « Estimating the true global burden of mental illness », Lancet Psychiatry , 3, p. 171-178.

Wang S., 2019, « Les nouvelles circulations de la médecine chinoise : après l'Afrique, l'Europe », Mouvements , 2, 98, p. 133-141.

Who, 2015, « Mental heath atlas 2014 », Geneva, World Health Organization.

Wone I., 2018, « Les défis de l'efficacité dans la planification des systèmes de santé en Afrique de l'Ouest ", Santé publique , 30 , 6, p. 905-909.

\section{NOTES}

1. Cet état des lieux statistiques contribue à invisibiliser la question de la santé mentale comme enjeu de santé publique comme en témoigne la dernière Enquête Continue sur la Prestation des Services de Soins de Santé, sur les 529 pages de ce rapport, pas une seule ligne est consacrée à la santé mentale (Agence Nationale de la Statistique et de la Démographie (ANSD) [Sénégal], et ICF. 2018. Sénégal : Enquête Continue sur la Prestation des Services de Soins de Santé (ECPSS) 2017 . Dakar, Sénégal, et Rockville, Maryland, USA : ANSD et ICF).

2. Le pénc est une réunion regroupant de manière hebdomadaire les soignants, les accompagnants et les personnes malades. Durant cette séance collective les patients partagent leurs expériences et s'expriment librement sur le vécu de leur hospitalisation, les effets de leur traitement et l'évolution de la maladie. 
3. L'hospitalisation et l'internement sont régis par la loi $n{ }^{\circ}$ 75-80 du 9 juillet 1975 relative au traitement des maladies mentales et au régime d'internement de certaines catégories d'aliénés, législation héritée de la loi française de 1838.

4. En 2007, la part de la population couverte pour le risque maladie tous régimes confondus était d'environ 20\%. En 2008, est lancée la Stratégie d'Extension de la Couverture du Risque Maladie des Sénégalais. Elle se décline en trois axes: amélioration et extension des initiatives de gratuité, réforme de l'assurance maladie obligatoire, stratégie de développement des mutuelles de santé. Ces actions ne se concrétisent réellement qu'à partir de 2013 dans le cadre du Plan de développement de la Couverture Maladie Universelle lancé par Macky Sall (2013-2017) qui vise en particulier les personnes du secteur informel et du monde rural. En 2015, est créée l'Agence de la Couverture Maladie Universelle afin d'accélérer l'extension de la couverture maladie. En 2017, le taux de couverture tous régimes confondus est estimé à $47 \%$ et le taux de pénétration des mutuelles à $37 \%$. Ces résultats positifs ne doivent pas obérer certains problèmes comme par exemple le taux de survie des mutuelles, le taux de recouvrement, l'information des populations en milieu rural (Deville C., Hane F., Ridde V., Touré L., « La Couverture universelle en santé au Sahel : la situation au Mali et au Sénégal en 2018 », Working Paper du Ceped, n 40, Ceped - UMR 196 Université Paris Descartes IRD -, Paris, 2018.

5. Différentes catégories d'esprits (génies, esprits ancestraux, seytan, djinn) dont les rabs wolof-lébou sont évoqués dans les pratiques magico-thérapeutiques. Le culte des rabs a été étudié en particulier par Andras Zempléni, « La Dimension thérapeutique du culte des Rab, Ndöp, Tuuru et Samp. Rites de possession chez les Lebou et les Wolof ", Psychopathologie africaine , 1966, 2 (3) : 295-439.

6. Di Méo (2006) renvoie au concept de territorialité définit par Claude Raffestin (1986) : "La territorialité reflète la multi dimensionnalité du vécu territorial des membres d'une collectivité ». Même si elle agrège, pour chacun, la représentation d'espaces socialement et collectivement repérés, qualifiés et partagés, elle concerne, d'abord, le sujet humain. Elle relève de sa sensibilité, de sa logique, de ses capacités réflexives et imaginatives personnelles. Elle se calque sur son espace vécu et en façonne l'unité. Elle s'enrichit de ses expériences, de ses apprentissages sociaux permanents. La territorialité agence et articule les échelles des territoires et des réseaux que fréquente chaque individu.

7. Le terme de « médecine prophétique » renvoie à la médecine tirée de l'islam, tandis que l'expression «médecine mystique " se réfère aux pratiques magico-religieuses impliquant l'action des génies, d'esprits ou des divinités dans la délivrance des maux et des souffrances.

8. Henri Collomb (1913-1979) psychiatre militaire français. Il développe une nouvelle approche de la psychiatrie en réaction à la psychiatrie coloniale en cherchant à prendre en compte le contexte culturel et social des populations. Arrivé en 1958 au Sénégal, il occupe la chaire de neuropsychiatrie, et développe à l'hôpital de Fann une nouvelle école de psychiatrie (école de Fann ou de Dakar) en réunissant autour de lui, outre une équipe médicale, des anthropologues et sociologues, et inaugura une collaboration avec des guérisseurs.

9. La population du Sénégal compte $96,1 \%$ de musulmans qui se répartissent au sein de plusieurs confréries dont celle des Mourides. Ceux-ci constituent la seconde confrérie du pays et représentent presque $32 \%$ de la population ( http://anads.ansd.sn/ 
index.php/catalog/9/datafile/F7/V409 consulté le 17 octobre 2019). Fondé par Cheikh Ahmadou Bamba, le mouridisme s'inscrit dans un islam sunnite et soufi. Selon son enseignement, les individus doivent être indépendants économiquement afin de pourvoir vivre leur foi et assurer la vie de leur famille et de la communauté musulmane. L'activité économique doit permettre aux disciples de se consacrer au travail spirituel afin de se rapprocher de Dieu. A l'origine très impliqués dans l'économie de l'arachide, les Mourides ont su diversifier leurs activités entrepreneuriales, leurs investissements, en contribuant notamment au développement des réseaux migratoires. Leur devise "travaille comme si tu ne devais jamais mourir, et prie comme si tu devais mourir demain » résume cette éthique du travail.

10. Le terme de daara (mot arabe passé dans le langage courant) désigne au Sénégal les écoles coraniques.

11. L'artane ${ }^{\circledast}$ est prescrit contre les effets indésirables des traitements neuroleptiques depuis le début des années 1990 et dans le traitement de la maladie de Parkinson. Parmi les effets secondaires, on note la confusion des idées, les troubles de l'accommodation, sécheresse de la bouche, difficulté à uriner, constipation.

12. La rispéridone est antipsychotique de la deuxième génération, utilisé dans le traitement de la schizophrénie, les épisodes maniaques, associés à des troubles bipolaires, troubles des conduites. Il est commercialisé sous le nom de Rispéridal ${ }^{\circledR}$. Ses effets indésirables peuvent être la sédation/somnolence, retrait émotionnel, céphalée, troubles de la libido.

13. Plus rarement ont également été évoqués dans des entretiens le valium ${ }^{\circledast}$ et le tranxene $e^{\oplus}$, qui appartiennent comme le lexomil ${ }^{\circledR}$ a la classe des benzodiazepines. Ces anxiolytiques (tranquillisants) sont utilisés dans le traitement des manifestations anxieuses sévères et/ou invalidantes. Ils peuvent avoir notamment comme effets indésirables des troubles de l'attention, des troubles de la parole, de la cognition, de l'insomnie, des cauchemars, une dépendance physique et psychologique.

14. Le Sénégal, tout en demeurant un pays de transit et de consommation, est récemment devenu un pays producteur de cannabis (voir OCDE, Flux financiers illicites. L'économie du commerce illicite en Afrique de l'Ouest . Paris, OCDE, 2018).

\section{RÉSUMÉS}

A partir de données anthropologiques et sociodémographiques collectées au Sénégal auprès de différentes catégories d'acteurs impliquées dans les quêtes thérapeutiques qu'engagent des personnes malades, seules ou soutenues par « leur(s) accompagnant(s) » afin de remédier à une pathologie mentale, sont décrites diverses circulations qui contribuent à structurer des parcours thérapeutiques souvent longs et chaotiques avant d'accéder à des soins psychiatriques. Les circulations des acteurs, des produits et des pratiques sont analysées à différentes échelles et interprétées par rapport à différents facteurs: l'inégale répartition territoriale de l'offre psychiatrique, les représentations des troubles psychiques et de leur guérison qui les 
rapprochent des maladies chroniques dans un contexte de pluralisme thérapeutique et de stigmatisation, certaines pratiques d'automédication au sein des familles en réponse au coût des soins en l'absence de couverture médicale universelle généralisée, enfin l'émergence de discours «psy » au sein de la société sénégalaise.

In this article we have chosen to focus on the circulations related to therapeutic mental health quests in the countries of the South through the example of Senegal. Mental health is not one of the priority health programs supported by the Global Fund, few countries in the South have specific mental health policies and programs, and it is not the subject of strong political and financial mobilization. According to WHO estimates, the majority of people with severe mental disorders are untreated and have diminished rights. With more accurate measurement of the disease burden, mental health has been included in the objectives of sustainable development and in overall public health. It is, in addition, a development issue given its social and economic costs to countries and families. Nevertheless, research on mental health in sub-Saharan Africa, whatever the discipline, is still too rare.

Senegal was chosen for this research for two reasons: first, since French colonization, it has had an internationally recognized psychiatric range of services, and second Senegalese society is characterized by international migration and significant circular mobility, which anchors it in processes of globalization. The various circulations that contribute to structuring therapeutic pathways that are often long and chaotic before accessing psychiatric care are described mainly on the basis of anthropological data collected from people experiencing mental illness, either alone or supported by "their accompanying person(s)". These data are based on field surveys conducted in several psychiatric services and mental health centers throughout the country. Consultations were followed in agreement with the medical and administrative authorities, observations and interviews were carried out with healers, sick people and their "companions" in order to understand the therapeutic pathways, the management of the disease and drug treatments as well as the representations of psychological disorders in a context of stigmatization of madness. The investigation continued beyond the walls of the psychiatric institution with healers, marabouts and representatives of mystical medicine in order to take into account the plurality of therapeutic remedies and the movements that take place at local, national and even transnational levels. The interviews were conducted either in French or Wolof (translated by an investigator trained in health anthropology).

The circulation of actors, products and practices is analyzed at different spatial and social scales and interpreted in relation to different explanatory and contextual factors. In the Senegalese health care system, mental health is not included in the primary care system. Map 1 reveals an insufficient supply of psychiatric care and deep inequalities between the West (the capital Dakar region) and the East of the country, as with other medical disciplines that are under-staffed and under-resourced. Sick people who consult in health centers or dispensaries are referred to the psychiatric unit in the health region where they reside or to the private sector if they have sufficient resources. Nevertheless, the pyramidal medical hierarchy is not always respected because it goes against the social logic of patients and their families who wish to consult in the greatest secrecy because of the social representations of madness. Therapeutic pathways therefore depend in part on accessibility and the additional costs of distance.

The accounts of the therapeutic pathways reveal that psychiatry is often considered the ultimate solution in the event of an acute crisis, particularly in rural areas. It is a solution sometimes forced by the neighborhood or the authorities in the face of symptoms that cause fear and disapproval. Given the context of stigmatization and reluctance towards psychiatric institutions, families are experimenting with other therapeutic alternatives with healers and marabouts who may specialize in the treatment of madness or in a so-called "mystical" medicine that is rooted in Islam. This is also due to certain popular beliefs that insanity is not curable, which hinders 
adherence to psychiatric treatment. This leads to the interruption of treatment as soon as the patient is perceived to be doing better. The narratives of the interviewees also reveal selfmedication practices that aim to regulate the behavior of sick people in the family and social space of the community. It helps to limit medical and indirect costs related to accessibility, as many families do not have universal medical coverage or sufficient financial resources. It reveals the circulation and sometimes diverted use of medicines after a medical check-up. In conclusion, we observe that the question of the management of psychological disorders is similar to that of chronic diseases (adherence to treatment, representation of recovery). It is also part of a context where a plurality of actors are taking hold of a protean "psy" discourse within Senegalese society.

INDEX

Mots-clés : santé mentale, quête thérapeutique

Index géographique : Sénégal

\section{AUTEUR}

VÉRONIQUE PETIT

Socio-démographe, Professeur Université Paris Descartes, CEPED/IRD 